TRANSACTIONS OF THE

AMERICAN MATHEMATICAL SOCIETY

Volume 359, Number 5, May 2007, Pages 2361-2381

S 0002-9947(06)04041-4

Article electronically published on June 13, 2006

\title{
RIGIDITY OF SMOOTH SCHUBERT VARIETIES IN HERMITIAN SYMMETRIC SPACES
}

\author{
JAEHYUN HONG
}

\begin{abstract}
In this paper we study the space $\mathcal{Z}_{k}\left(G / P, r\left[X_{w}\right]\right)$ of effective $k$ cycles $X$ in $G / P$ with the homology class equal to an integral multiple of the homology class of Schubert variety $X_{w}$ of type $w$. When $X_{w}$ is a proper linear subspace $\mathbb{P}^{k}(k<n)$ of a linear space $\mathbb{P}^{n}$ in $G / P \subset \mathbb{P}(V)$, we know that $\mathcal{Z}_{k}\left(\mathbb{P}^{n}, r\left[\mathbb{P}^{k}\right]\right)$ is already complicated. We will show that for a smooth Schubert variety $X_{w}$ in a Hermitian symmetric space, any irreducible subvariety $X$ with the homology class $[X]=r\left[X_{w}\right], r \in \mathbb{Z}$, is again a Schubert variety of type $w$, unless $X_{w}$ is a non-maximal linear space. In particular, any local deformation of such a smooth Schubert variety in Hermitian symmetric space $G / P$ is obtained by the action of the Lie group $G$.
\end{abstract}

\section{INTRODUCTION}

In this paper we study the space $\mathcal{Z}_{k}\left(G / P, r\left[X_{w}\right]\right)$ of effective $k$-cycles $X$ in $G / P$ with the homology class equal to an integral multiple of the homology class of a Schubert variety $X_{w}$ of type $w$. For example, $\mathcal{Z}_{1}\left(\mathbb{P}^{n},\left[\mathbb{P}^{1}\right]\right)$ consists of all $\mathbb{P}^{1}$ 's in $\mathbb{P}^{n}$ so every element in $\mathcal{Z}_{1}\left(\mathbb{P}^{n},\left[\mathbb{P}^{1}\right]\right)$ is obtained by acting $g \in S L(n+1)$ on a fixed $\mathbb{P}^{1}$. However, $\mathcal{Z}_{1}\left(\mathbb{P}^{n}, r\left[\mathbb{P}^{1}\right]\right), r>1$, contains not only the sum $\mathbb{P}^{1}+\cdots+\mathbb{P}^{1}$ of $r \mathbb{P}^{1}$ 's but also the sum $C_{1}+\cdots+C_{s}$ of curves $C_{i}$ of degree $r_{i}$ in $\mathbb{P}^{n}$, where $r_{1}+\cdots+r_{s}=r$.

In general the cycle space $\mathcal{Z}_{k}(M, z)$ for $z \in H_{2 k}(M, \mathbb{Z})$ is complicated. But, as in the case of $\mathbb{P}^{1}$ in $\mathbb{P}^{n}$, for certain subvarieties $X$ of a homogeneous space $G / P$, the cycle space $Z_{k}(G / P,[X])$ is rather simple: $G$ acts on $\mathcal{Z}_{k}(G / P,[X])$ transitively. In particular, any local deformation of $X$ in $G / P$ is given by the action of $G$. Even more, for certain Schubert varieties $X_{w}$ in a Hermitian symmetric space $G / P$, the cycle space $\mathcal{Z}_{k}\left(G / P, r\left[X_{w}\right]\right)$ consists of the sums of $r$ Schubert varieties of type $w$ and is just the $r$-symmetric product of the $G$-homogenous space $\mathcal{Z}_{k}\left(G / P,\left[X_{w}\right]\right)$.

Question. For which Schubert variety $X_{w}$ in Hermitian symmetric space $G / P$ does the cycle space $\mathcal{Z}_{k}\left(G / P, r\left[X_{w}\right]\right)$ consist of the sums of $r$ Schubert varieties of type $w$ ?

Walters and Bryant studied this problem by transforming it to the problem on the integral varieties of differential systems $([\mathrm{W},[\mathrm{B}])$. From the fact that there exists a closed positive differential form $\phi_{w}$ for $w \in W^{P}$ such that $\int_{X_{w}} \phi_{v}=0$ for

Received by the editors October 26, 2004 and, in revised form, April 13, 2005.

2000 Mathematics Subject Classification. Primary 14C25, 32M15, 14M15.

Key words and phrases. Analytic cycles, Hermitian symmetric spaces, Schubert varieties.

(C)2006 American Mathematical Society Reverts to public domain 28 years from publication 
all $v \neq w$ with $\ell(v)=\ell(w)(\underline{\mathrm{K} 2}])$, it follows that

$$
\begin{aligned}
{[X]=r\left[X_{w}\right] } & \Leftrightarrow \int_{X} \phi_{v}=0, \forall v \neq w, \ell(v)=\ell(w) \\
& \left.\Leftrightarrow \phi_{v}\right|_{X}=0, \forall v \neq w, \ell(v)=\ell(w) .
\end{aligned}
$$

Therefore, such an $X$ satisfies a first order holomorphic partial differential equation. In particular, if $X$ is tangent to a Schubert variety of type $w$ at each point $x \in X$, which may depend on the point $x$, then we have $[X]=r\left[X_{w}\right]$ for some $r \in \mathbb{Z}$. Since the ray generated by the Schubert cycle $X_{w}$ is extremal, if an effective cycle $X=X_{1}+\cdots+X_{n}$ has the homology class $[X]=r\left[X_{w}\right]$, then $r=r_{1}+\cdots+r_{n}$ and $\left[X_{i}\right]=r_{i}\left[X_{w}\right]$, where $X_{i}$ is an irreducible compact complex variety of dimension $k$. So we may assume that $X$ is irreducible.

Define a differential system $\mathcal{R}_{w}$ by putting together all tangent $\ell(w)$-subspaces $W$ such that $\left.\phi_{v}\right|_{W}=0$ for all $v \neq w$ with $\ell(v)=\ell(w)$. We say that the Schubert variety $X_{w}$ is Schur rigid if any irreducible integral variety of $\mathcal{R}_{w}$ is a Schubert variety of type $w$, or equivalently, $\mathcal{Z}_{k}\left(G / P, r\left[X_{w}\right]\right)$ consists only of the sums of Schubert cycles of type $w$. Now the question becomes how to compute $\mathcal{R}_{w}$ and how to find all the integral varieties of $\mathcal{R}_{w}$.

The differential system $\mathcal{R}_{w}$ contains the differential system $\mathcal{B}_{w}$ with the fiber consisting of the tangent space of all the Schubert varieties $X_{w}$ of type $w$ passing through the given point. We say that the Schubert variety $X_{w}$ is Schubert rigid if any irreducible integral variety of $\mathcal{B}_{w}$ is a Schubert variety of type $w$. Thus $X_{w}$ is Schur rigid if $\mathcal{B}_{w}$ is equal to $\mathcal{R}_{w}$ and $X_{w}$ is Schubert rigid ([W], Section 2.8 of [B]). Conversely, $X_{w}$ is not Schur rigid if either $\mathcal{B}_{w}$ is a proper subvariety of $\mathcal{R}_{w}$ or $\mathcal{B}_{w}$ is not Schubert rigid (Proposition 2 of [B], Proposition 2.10).

It is known that smooth Schubert varieties in Grassmannian $\operatorname{Gr}(m, n)$ are Schubert rigid unless it is a non-maximal linear space in $\operatorname{Gr}(m, n)([\mathrm{W}])$. Schur and Schubert rigidity of several kinds of smooth and singular Schubert varieties in Hermitian symmetric spaces are investigated systematically in $[\mathrm{B}$. The Schubert rigidity of linear spaces in general homogeneous spaces with $b_{2}=1$ is studied in $[\mathrm{CH}]$.

In this paper we restrict ourselves to the case of smooth Schubert varieties $X_{w}$ in Hermitian symmetric spaces $G / P$. Since the homology space $H_{2 k}\left(Q^{2 n-1}, \mathbb{Z}\right)$ of the quadric $Q^{2 n-1}$ of odd dimension is $\mathbb{Z}\left[X_{w}\right]$, any subvariety of dimension $k$ has homology class $r\left[X_{w}\right]$ for some $r \in \mathbb{Z}$. So no Schubert variety in $Q^{2 n-1}$ is Schur rigid. If $X_{w}$ is a proper linear subspace $\mathbb{P}^{k}(k<n)$ of a linear space $\mathbb{P}^{n}$ contained in $G / P$, then $\mathcal{Z}_{k}\left(G / P, r\left[X_{w}\right]\right)$ contains $\mathcal{Z}_{k}\left(\mathbb{P}^{n}, r\left[\mathbb{P}^{k}\right]\right)$, so $X_{w}$ is not Schur rigid.

When $X_{w}$ is a maximal linear space in Hermitian symmetric space $G / P$ of classical type or is a sub-Lagrangian Grassmannian $L_{m-a}$ in the Lagrangian Grassmannian $L_{m}$, the Schur rigidity of $X_{w}$ is proved in B (Theorems 2, 5, 14, 15, 18 of [B]). Generalizing this result, we prove that, with the above trivial exceptions, smooth Schubert varieties are Schur rigid.

Main Theorem. Let $G / P$ be a Hermitian symmetric space other than an odddimensional quadric. Then any smooth Schubert variety $X_{w}$ in $G / P$ is Schur rigid except when $X_{w}$ is a non-maximal linear space in $G / P$. Here, we consider $G / P$ as a projective variety by the minimal equivariant embedding $G / P \subset \mathbb{P}(V)$.

After giving some preliminaries on Schubert varieties in Section 2, we find a criterion that is used for determining the Schubert rigidity of a Schubert variety $X_{w}$ and one for the equality $\mathcal{B}_{w}=\mathcal{R}_{w}$ in Section 3 . 
To prove the Schubert rigidity, we use the result of Goncharov on the integral varieties of $F$-structures ([G]). The fiber $B_{w}$ of $\mathcal{B}_{w}$ is an orbit of the semisimple part of $P$, which can be considered as a subgroup of $G L(k, \mathfrak{m})$, where $\mathfrak{m} \simeq T_{0}(G / P)$. In this case, Goncharov showed that if the cohomology space $H^{1,1}\left(B_{w}\right)$ vanishes, then there is only one integral variety of $\mathcal{B}_{w}$ passing through a fixed point and tangent to a fixed tangent subspace. Thus $X_{w}$ is Schubert rigid if $H^{1,1}\left(B_{w}\right)$ vanishes (Section 3.1).

For the equality $B_{w}=R_{w} \subset G r(k, \mathfrak{m})$ of the fibers of $\mathcal{B}_{w}$ and $\mathcal{R}_{w}$ at $o \in G / P$, we consider the decomposition $\bigwedge^{k} \mathfrak{m}=\bigoplus_{\ell(w)=k} \mathbf{I}_{w}$ of the $k$-wedge product of the tangent space $T_{o}(G / P) \simeq \mathfrak{m}$ as the direct sum of irreducible representation spaces of the semisimple part of $P(\overline{\mathrm{K} 2})$ and observe that $R_{w}$ is given by the intersection $\operatorname{Gr}(k, \mathfrak{m}) \cap \mathbb{P}\left(\mathbf{I}_{w}\right)$ and that $B_{w}$ is the highest weight orbit in $\mathbb{P}\left(\mathbf{I}_{w}\right)$. Thus if the complement of tangent space $T_{\left[\mathfrak{n}_{w}\right]} B_{w}$ in $T_{\left[\mathfrak{n}_{w]}\right.} G r(k, \mathfrak{m})$ intersects the tangent space $T_{\left[\wedge^{k} \mathfrak{n}_{w}\right]} \mathbb{P}\left(\mathbf{I}_{w}\right)$ trivially, then $B_{w}$ equals $R_{w}$ (Section 3.2 ).

To compute the cohomology space $H^{1,1}\left(B_{w}\right)$ and the complement of $T_{\left[\mathfrak{n}_{w}\right]} B_{w}$, we use the fact that a smooth Schubert variety $X_{w}$ in a Hermitian symmetric space corresponds to a subdiagram $\delta$ of the Dynkin diagram of $G$ ([BP], [LW], Proposition 2.12). Then we can apply the theory of Lie algebra cohomology developed by Kostant ([K1]) to compute them (Proposition 3.7 and Proposition 3.8).

In Section 4, we verify these conditions and prove the Main Theorem. Furthermore, we prove the Schubert rigidity of smooth Schubert varieties in general homogeneous space in the same way under some assumptions (Proposition 4.4).

Our representation-theoretic method of proving the equality $B_{w}=R_{w}$ is new and can be applied to the case of singular Schubert varieties in general, because the assumption that $X_{w}$ is smooth is used only for computing the complement of $T_{o} B_{w}$ in $T_{o} G r(k, \mathfrak{m})$ in a uniform way. We expect that the rigidity of smooth Schubert varieties will serve as the building blocks for proving the rigidity of singular Schubert varieties in general.

\section{Differential Systems}

2.1. Schubert varieties. Let $\mathfrak{g}$ be a complex simple Lie algebra. Choose a Cartan subalgebra $\mathfrak{h}$ of $\mathfrak{g}$. Let $\Delta$ be the set of all roots of $\mathfrak{g}$ with respect to $\mathfrak{h}$. Fix a system $\mathcal{S}=\left\{\alpha_{1}, \cdots, \alpha_{n}\right\}$ of simple roots of $\mathfrak{g}$. Let $\Delta^{+}$be the set of positive roots with respect to $\mathcal{S}$.

For a subset $I$ of $\mathcal{S}$, set $\Delta_{I}=\Delta \cap \mathbb{Z} I$, and let $\mathfrak{p}_{I}$ be the parabolic subalgebra generated by $I$, that is, $\mathfrak{p}_{I}=\mathfrak{p}_{0}+\mathfrak{m}^{*}$, where $\mathfrak{p}_{0}:=\mathfrak{h}+\sum_{\alpha \in \Delta_{I}} \mathfrak{g}_{\alpha}$ is the reductive part and $\mathfrak{m}^{*}:=\sum_{\alpha \in \Delta^{+} \backslash \Delta_{I}}$ is the nilpotent part. Then we have $\mathfrak{g}=\mathfrak{p}_{I}+\mathfrak{m}$, where $\mathfrak{m}=\sum_{\alpha \in \Delta^{-} \backslash \Delta_{I}}$. The empty set $I=\emptyset$ corresponds to the Borel subalgebra $\mathfrak{b}=\mathfrak{h}+\sum_{\alpha \in \Delta^{+}} \mathfrak{g}_{\alpha}$. Set $\Delta\left(\mathfrak{m}^{*}\right)=\Delta^{+} \backslash \Delta_{I}$.

Let $W$ be the Weyl group of $\mathfrak{g}$. For each $w \in W$, define a subset $\Delta(w)$ of $\Delta^{+}$by $\Delta(w):=w \Delta^{-} \cap \Delta^{+}$. Then the number $|\Delta(w)|$ of elements in $\Delta(w)$ is equal to the length $\ell(w)$ of $w$. The subset $W^{I}$ of $W$ defined by $W^{I}=\left\{w \in W: \Delta(w) \subset \Delta\left(\mathfrak{m}^{*}\right)\right\}$ is equal to $\left\{w \in W: w^{-1}\left(\Delta_{I}^{+}\right) \subset \Delta^{+}\right\}$.

Define a dual set of weights by requiring $\left\langle\lambda_{i}, \alpha_{j}^{\vee}\right\rangle=\delta_{i, j}$, where $\alpha_{j}^{\vee}=\frac{2 \alpha_{j}}{\left\langle\alpha_{j}, \alpha_{j}\right\rangle}$. Set $\rho=\frac{1}{2} \sum_{\alpha \in \Delta^{+}} \alpha=\sum_{i} \lambda_{i}$ and put $\rho_{I}=\sum_{\alpha_{i} \in \mathcal{S} \backslash I} \lambda_{i}$. Then $W$ (respectively, $W^{I}=W / W_{I}$ ) is bijective to the orbit of $\rho$ (respectively, $\rho_{I}$ ) under the action of $W$ given by $\rho \rightarrow w^{-1} \rho$ ([K2, $\left.\left.\mathrm{BE}\right]\right)$. 
Let $G$ be a connected Lie group with Lie algebra $\mathfrak{g}$ and let $P_{I}, B$ and $H$ be Lie subgroups of $G$ corresponding to $\mathfrak{p}_{I}, \mathfrak{b}$ and $\mathfrak{h}$. If $P=P_{I}$, then $\Delta_{I}, W^{I}, \rho_{I}$ are also denoted by $\Delta_{P}, W^{P}, \rho_{P}$, respectively.

The Weyl group $W$ of $\mathfrak{g}$ is isomorphic to the quotient $N_{G}(H) / H$ of the normalizer of $H$ by $H$. So we may consider $w \in W$ as an element in $G$. In the same way, if $U$ is a real form of $G$, we may take $w$ in $U$, too. Let $o$ be the origin of $G / P$. For each $w \in W^{P}$, considering $w$ as an element of $G$, let $V_{w}$ be the $B$-orbit $V_{w}=B w^{-1} \cdot o$. Then $G / P$ is decomposed as a disjoint union $\bigcup_{w \in W^{P}} V_{w}$ of $B$-orbits (Section 6 of [K2]).

For $w \in W$, let $\mathfrak{n}_{w}^{*}$ (respectively, $\mathfrak{n}_{w}$ ) be the nilpotent Lie subalgebra spanned by the root vectors in $\Delta(w)$ (respectively, $-\Delta(w)$ ), and let $N_{w}^{*}$ and $N_{w}$ be the subgroups corresponding to $\mathfrak{n}_{w}^{*}$ and $\mathfrak{n}_{w}$. Since $w \Delta\left(w^{-1}\right)=-\Delta(w), N_{w^{-1}}^{*}$ and $N_{w}$ are conjugate by an element in $G$, that is, $w N_{w^{-1}}^{*} w^{-1}=N_{w}$.

Write $B=N^{*} H$, where $N^{*}$ is the unipotent part of $B$. Let $\kappa$ be the elements in $W$ of maximal length. Then $\kappa \Delta^{-}=\Delta^{+}$. The isotropy group of $N^{*}$ at $w^{-1} \cdot o$ is $N_{w^{-1} \kappa}^{*}$, and $V_{w}$ is isomorphic to the $N_{w^{-}}$orbit $N_{w} \cdot o$ at the origin by the action of an element $w \in G$, that is, $w V_{w}=w N_{w^{-1}}^{*} w^{-1} \cdot o=N_{w} \cdot o$ (Section 6 of [K2]). The closure $X_{w}$ of $V_{w}$ is an irreducible subvariety of dimension $\ell(w)$. We call $X_{w}$ a Schubert variety of type $w$.

Proposition 2.1. Let $G / P$ be a Hermitian symmetric space. Let $U$ be a compact real form of $G$. Write $\mathfrak{p}=\mathfrak{p}_{0}+\mathfrak{m}^{*}$ and $\mathfrak{g}=\mathfrak{p}+\mathfrak{m}$, where $\mathfrak{p}_{0}$ is the reductive part and $\mathfrak{m}^{*}$ is the nilpotent part. Then

(1) $\bigwedge^{k} \mathfrak{m}$ is decomposed as the direct sum $\bigoplus_{w \in W^{P}(k)}(\bigwedge \mathfrak{m})^{w \rho-\rho}$ of irreducible $\mathfrak{p}_{0}$-representation spaces $\mathbf{I}_{w}:=(\bigwedge \mathfrak{m})^{w \rho-\rho}$, where $W^{P}(k)=\left\{w \in W^{P}: \ell(w)=k\right\}$. The highest weight vector in $\mathbf{I}_{w}$ is the decomposable $\ell(w)$-vector $e_{-\Delta(w)}$, the wedge product of root vectors $x_{\alpha}$ of roots $\alpha \in-\Delta(w)$.

(2) For $w \in W^{P}$, define $\phi_{w}$ by the $U$-invariant differential $(k, k)$-form which is given by $(\sqrt{-1})^{k^{2}} \sum_{i} \zeta_{i} \wedge \bar{\zeta}_{i}$ at the origin, where $\left\{\zeta_{i}\right\}$ be an orthonormal basis of $\mathbf{I}_{w}^{*}$. Then $\phi_{w}$ is closed and positive and satisfies

$$
\left\langle\left[\phi_{v}\right],\left[X_{w}\right]\right\rangle=\int_{X_{w}} \phi_{v}=0, \text { for } v \neq w .
$$

Proof. (1) Theorem 5.14 or Corollary 8.2 of [K1].

(2) Section 5.6, Corollary 5.4 and Corollary 6.15 of [K2]. To see it more directly, note that $w V_{w}=N_{w} \cdot o$ and that we can take $w$ in $U$. Since $\phi_{v}$ is $U$-invariant, we have $\int_{V_{w}} \phi_{v}=\int_{N_{w} \cdot o} \phi_{v}=0$.

Remark 2.2. Schubert varieties $X_{w}$ and closed differential forms $\phi_{w}$ such that $\left.\phi_{v}\right|_{X_{w}}=0$ for $v \neq w$ are defined on general homogeneous space $G / P$ (Corollary 6.15 of [K2] $)$. But, in general, $\phi_{w}$ is not of the form as in Proposition 2.1 and is more complicated.

Example 2.3. Let $G r(m, n)$ be the Grassmannian of $m$-dimensional subspaces of $\mathbb{C}^{n}$. For $\mathbf{a} \in P(m, n)=\left\{\mathbf{a}=\left(a_{1}, \cdots, a_{m}\right): n-m \geq a_{1} \geq \cdots \geq a_{m}\right\}$, the Schubert variety $\sigma_{\mathbf{a}}$ of type $\mathbf{a}$ is defined by the set

$$
\left\{E \in G r(m, n) \mid \operatorname{dim}\left(E \cap \mathbb{C}^{n-m+i-a_{i}}\right) \geq i\right\} .
$$

$\sigma_{\mathbf{a}}$ is a subvariety of $\operatorname{Gr}(m, n)$ of codimension $|\mathbf{a}|:=a_{1}+\cdots+a_{m}$. 
For $\mathbf{a} \in P(m, n)$, define its dual $\mathbf{a}^{*}$ by

$$
\mathbf{a}^{*}=\left(n-m-a_{m}, \cdots, n-m-a_{1}\right)
$$

and define its conjugate $\mathbf{a}^{\prime}=\left(a_{1}^{\prime}, \cdots, a_{n-m}^{\prime}\right)$ by

$$
\mathbf{a}_{i}^{\prime}=\sharp\left\{j \mid a_{j} \geq i\right\} \text { for } 1 \leq i \leq n-m .
$$

Let $E \in G r(m, n)$ and $Q$ be the quotient $V / E$. The $k$-th wedge product of $T_{E}^{*}(G r(m, n))=E \otimes Q^{*}$ is decomposed as the direct sum of irreducible $S L(E) \times$ $S L\left(Q^{*}\right)$ representation spaces as follows (Exercise 6.11 of $[\mathrm{FH}]$ ):

$$
\bigwedge^{k}\left(E \otimes Q^{*}\right)=\bigoplus_{|\mathbf{a}|=k} \mathbb{S}_{\mathbf{a}}(E) \otimes \mathbb{S}_{\mathbf{a}^{\prime}}\left(Q^{*}\right)
$$

where $\mathbb{S}_{\mathbf{a}}$ is the Schur functor of type a. Locally, $\phi_{\mathbf{a}}$ can be written as the sum $(\sqrt{-1})^{k^{2}} \sum_{i} \zeta_{i} \wedge \bar{\zeta}_{i}$, where $\left\{\zeta_{i}\right\}$ is an orthonormal basis of $\mathbb{S}_{\mathbf{a}}(E) \otimes \mathbb{S}_{\mathbf{a}^{\prime}}\left(Q^{*}\right)$.

We will explain how to construct the Schur module (or Weyl module) $\mathbb{S}_{\mathbf{a}}(V)$ for a vector space $V$ in Section 4, where we will prove the Schur rigidity.

\subsection{Schur and Schubert differential systems.}

Definition 2.4. Let $M$ be a manifold and let $G r(k, T M)$ be the Grassmannian bundle of $k$-subspaces of the tangent bundle $T M$. A subvariety $\mathcal{F}$ of $G r(k, T M)$ is called a differential system on $M$. A subvariety $X$ of $M$ is said to be an integral variety of the differential system $\mathcal{F}$ if at each smooth point $x \in X$, the tangent space $T_{x} X$ is an element of the fiber $\mathcal{F}_{x}$. We say that $\mathcal{F}$ is integrable if at each point $x \in M$ and $y \in \mathcal{F}_{x}$ there is an integral variety passing through $x$ and tangent to the subspace $W_{y}$ of $T_{x} M$ corresponding to $y$.

In $\left[\mathrm{W}\right.$ ] and $\left[\mathrm{B}\right.$, they consider two differential systems $\mathcal{B}_{w}$ and $\mathcal{R}_{w}$ to solve the rigidity problem of the Schubert variety $X_{w}$. By taking a different Borel subgroup $B \subset P$, we get a family of Schubert varieties of type $w$. This family induces the first differential system.

Definition 2.5. Let $M=G / P$ be a Hermitian symmetric space. For each $w \in$ $W^{P}$, the Schubert differential system $\mathcal{B}_{w}$ of type $w$ is the differential system with the fiber consisting of the tangent spaces of all the Schubert varieties $X_{w}$ of type $w$ passing through the given point. We say that $X_{w}$ is Schubert rigid if Schubert varieties of type $w$ are the only irreducible integral varieties of $\mathcal{B}_{w}$.

Definition 2.6. Let $M=G / P$ be a Hermitian symmetric space. For each $w \in$ $W^{P}$, the Schur differential system $\mathcal{R}_{w}$ of type $w$ is the differential system with the fiber defined by the intersection

$$
\bigcap_{v \neq w, \ell(v)=\ell(w)} Z\left(\phi_{v}\right)
$$

where $Z\left(\phi_{v}\right)$ is the set of an $\ell(w)$-subspace of $T_{x} M$ on which $\phi_{v}$ vanishes. We say that $X_{w}$ is Schur rigid if Schubert varieties of type $w$ are the only irreducible integral varieties of $\mathcal{R}_{w}$.

Proposition 2.1 provides a way to transform the problem on the cycle space $\mathcal{Z}\left(G / P, r\left[X_{w}\right]\right)$ to the problem on the integral varieties of the differential system $\mathcal{R}_{w}$ (Section 2.8 .1 of $[\mathrm{B}],[\mathrm{W}]$ ). 
Proposition 2.7. Let $G / P$ be a Hermitian symmetric space. Take $w \in W^{P}$. For a subvariety $X$ of $G / P, X$ has the homology class $[X]=r\left[X_{w}\right]$ for an integer $r$ if and only if $X$ is an integral variety of $\mathcal{R}_{w}$. Therefore, the cycle space $\mathcal{Z}\left(G / P, r\left[X_{w}\right]\right)$ consists of formal sums of Schubert varieties of type $w$ if and only if $X_{w}$ is Schur rigid.

For $w \in W^{P}$, let $B_{w}$ (resp. $R_{w}$ ) be the fiber of $\mathcal{B}_{w}$ (resp. $\mathcal{R}_{w}$ ) at $o \in G / P$. Note that $B_{w}$ is closed and $R_{w}$ is connected (Remark 2 and Remark 12 of [B]). Proposition 2.1 gives a more refined structure of $R_{w}$ and $B_{w}$.

Proposition 2.8. For $w \in W^{P}$,

(1) $R_{w}$ is equal to the intersection

$$
G r(k, \mathfrak{m}) \cap \mathbb{P}\left(\mathbf{I}_{w}\right) \subset \mathbb{P}\left(\bigwedge^{k} \mathfrak{m}\right)
$$

and

(2) $B_{w}$ is the orbit of a highest weight vector in the irreducible representation space $\mathbb{P}\left(\mathbf{I}_{w}\right)$ of the reductive component $P_{0}$ of $P$.

Proof. (1) Consider a complex $k$-subspace $W$ of $\mathfrak{m} \simeq T_{x}(G / P)$ as a one-dimensional vector space $\bigwedge^{k} W$ in $\bigwedge^{k} \mathfrak{m}$ which is decomposed as $\bigoplus_{\ell(w)=k} \mathbf{I}_{w}$. By Proposition 2.1 $(2), \phi_{v}$ is given by the sum $(\sqrt{-1})^{k^{2}} \sum_{i} \xi_{i} \wedge \bar{\xi}_{i}$ at $x$, where $\left\{\xi_{i}\right\}$ is an orthonormal basis of $\mathbf{I}_{v}^{*}$. If $\phi_{v}$ vanishes on a complex $k$-subspace $W$ of $T_{x}(G / P) \simeq \mathfrak{m}$, then so does any $\xi_{i}$. Thus every vector in the vector space $\bigwedge^{k} W$ is annihilated by any dual vector in the space $\mathbf{I}_{v}^{*}$, so it is contained in the complement $\bigoplus_{\tau \neq v} \mathbf{I}_{\tau}$ of $\mathbf{I}_{v}$ in $\bigwedge^{k} \mathfrak{m}$. Therefore, we have

$$
Z\left(\phi_{v}\right)=\operatorname{Gr}(k, \mathfrak{m}) \cap \mathbb{P}\left(\bigoplus_{\tau \neq v, \ell(\tau)=k} \mathbf{I}_{\tau}\right),
$$

and hence the intersection $R_{w}=\bigcap_{v \neq w, \ell(v)=k} Z\left(\phi_{v}\right)$ is equal to $G r(k, \mathfrak{m}) \cap \mathbb{P}\left(\mathbf{I}_{w}\right)$.

(2) Use Proposition 2.1(1).

Remark 2.9. In $\mathrm{B}$, the author computes the integral elements of the exterior differential ideal $\mathcal{I}_{w}$ which is generated by the sections of the subbundle of $\bigwedge^{k} T^{*}(G / P)$ associated to the component $\mathbf{I}_{w}^{*}$ in $\bigwedge^{k} \mathfrak{m}^{*}$ and then computes

$$
R_{w}=\bigcap_{v \neq w, \ell(v)=\ell(w)} Z\left(\phi_{v}\right)
$$

by using the fact that $Z\left(\phi_{v}\right)$ is equal to the set of all $k$-dimensional integral elements of $\mathcal{I}_{v}$ (Lemma 1 in $[\mathrm{B}]$ ). Since $Z\left(\phi_{v}\right)$ is given by the intersection $\operatorname{Gr}(k, \mathfrak{m}) \cap$ $\mathbb{P}\left(\bigoplus_{\tau \neq v, \ell(\tau)=k} \mathbf{I}_{\tau}\right)$, computing it is much more difficult than computing just their intersection $R_{w}=G r(k, \mathfrak{m}) \cap \mathbb{P}\left(\mathbf{I}_{w}\right)$. He uses it to classify globally generated holomorphic vector bundles with certain Chern classes vanishing. In this paper we are interested only in the rigidity problem, so it is enough to consider the intersection $R_{w}=G r(k, \mathfrak{m}) \cap \mathbb{P}\left(\mathbf{I}_{w}\right)$.

Proposition 2.10. Let $G / P$ be a Hermitian symmetric space. Take $w \in W^{P}$. Then $X_{w}$ is Schur rigid if and only if $B_{w}$ is equal to $R_{w}$ and $X_{w}$ is Schubert rigid.

Proof. If $R_{w}$ is equal to $B_{w}$ and $X_{w}$ is Schubert rigid, then $X_{w}$ is Schur rigid by definition. If $X_{w}$ is not Schubert rigid, then $X_{w}$ is not Schur rigid because $B_{w}$ is a subvariety of $R_{w}$. 
Suppose that $B_{w}$ is not equal to $R_{w}$. Then we can find an irreducible subvariety of $G / P$ which is an integral variety of $\mathcal{R}_{w}$ but which is not an integral variety of $\mathcal{B}_{w}$ in the same way as Bryant in the case when $G / P$ is the Grassmannian $\operatorname{Gr}(m, n)$ (Remark 4 and Proposition 2 of [B] $)$. We will present the way to get such a subvariety for completeness.

For a subspace $A$ of $\mathfrak{m} \simeq T_{x}(G / P)$ with $[A] \in R_{w} \backslash B_{w}$, consider the closure $X_{A}=\operatorname{cl}(\exp (A) \cdot x)$ of the orbit of the unipotent group $\exp (A)$ in $G / P$. Since $\mathfrak{m}$ is an abelian Lie algebra, any subspace $A$ of $\mathfrak{m}$ is again an abelian Lie algebra and thus is a nilpotent subalgebra of $\mathfrak{m}$. So $\exp (A)$ is nothing but $I d+A$ if we consider it in the matrix algebra.

Then $\exp (A) \cdot x$ is Zariski open dense in its closure $X_{A}$, so $X_{A}$ is an irreducible algebraic variety of $G / P$. Since the form $\phi_{v}$ vanishes on $\exp (A) \cdot x$ for $v \neq w, X_{A}$ is an integral variety of $\mathcal{R}_{w}$. But its tangent space at $x$ is not contained in $B_{w}$, so $X_{w}$ is not a Schubert variety of type $w$. Hence $X_{w}$ is not Schur rigid.

2.3. Smooth Schubert varieties. Let $\mathcal{D}(G)$ be the Dynkin diagram of $G$. Let $\gamma$ denote the simple root such that the complement $\mathcal{S} \backslash\{\gamma\}$ generates the parabolic subgroup $P$. Then the marked diagram $(\mathcal{D}(G), \gamma)$ represents the Hermitian symmetric space $G / P$.

To a connected subgraph $\delta$ of $(\mathcal{D}(G), \gamma)$ with $\gamma$ a node, we associate a smooth Schubert variety of type, say, $w \in W^{P}$. Here, $w$ is the longest element in the subgroup of the Weyl group of $G$ generated by the reflections by the simple roots in $\delta$ and $\Delta\left(w^{-1}\right)$ is equal to the set of all roots which are linear combinations of simple roots in $\delta$ with positive coefficients.

Conversely, we will show that in Hermitian symmetric space any smooth Schubert variety corresponds to a subgraph of the Dynkin diagram $\mathcal{D}(G)$ of $G$. To do this we need the following proposition on the singular locus of Schubert varieties in Hermitian symmetric spaces.

Proposition 2.11. Let $X_{w}$ be a Schubert variety in a Hermitian symmetric space $G / P$. Then the stabilizer $P_{w}$ in $G$ of $X_{w}$ is the parabolic subgroup generated by $\mathcal{S} \cap w^{-1}\left(\Delta_{P} \cup \Delta^{-}\right)$. Furthermore, $X_{w}$ is smooth if and only if $P_{w}$ acts on $X_{w}$ transitively.

Proof. Section 2.6, Proposition 3.3, and Proposition 4.4 of $\left[\mathrm{BP}\right.$. Note that $W^{P}$ in $\left[\mathrm{BP}\right.$ is the set of the inverse $w^{-1}$ of $w$ in $W^{P}$ in our paper.

Proposition 2.12. Let $G / P$ be a Hermitian symmetric space and let $(\mathcal{D}(G), \gamma)$ be the marked Dynkin diagram corresponding to $G / P$. Any smooth Schubert variety $X_{w}$ in $G / P$ corresponds to a connected subgraph of the Dynkin diagram $\mathcal{D}(G)$ with $\gamma$ a node.

Proof. Let $P_{w}=L_{w} U_{w}$ be the Levi decomposition of $P_{w}$, where $L_{w}$ is the Levi part and $U_{w}$ is the unipotent part. Then the semisimple part of $L_{w}$ has the simple root system $\mathcal{S} \cap w^{-1}\left(\Delta_{P} \cup \Delta^{-}\right)$and $U_{w}$ is generated by $U_{\alpha}$, for $\alpha \in \Delta^{+} \backslash w^{-1}\left(\Delta_{P} \cup \Delta^{-}\right)$.

The isotropy of $N^{*}$ at $w^{-1} \cdot o$ is $N_{w^{-1} \kappa}^{*}$ and $\Delta\left(w^{-1} \kappa\right)=\Delta^{+} \cap w^{-1} \kappa \Delta^{-}=$ $\Delta^{+} \cap w^{-1} \Delta^{+}$(Section 6 of [K2]). Thus $U_{w}$ and the semisimple Lie subgroup of $L_{w}$ corresponding to $\mathcal{S} \cap w^{-1}\left(\Delta_{P}^{+}\right)$act trivially on $w^{-1} \cdot o$. So $X_{w}$ corresponds to the subgraph $\delta$ of $\mathcal{D}(G)$ having $\mathcal{S} \cap w^{-1} \Delta^{-}$as the set of nodes.

For a classical group $G$, we can verify Proposition 2.12 case-by-case by using the description of smooth Schubert varieties in Section 5 of [LW]. 
Now consider $G / P$ as the orbit of the highest weight vector of the irreducible representation space $\mathbb{P}\left(V^{\rho_{P}}\right)$ with highest weight $\rho_{P}$. This is the minimal equivariant embedding of $G / P$ in the projective space. Then

(1) a subgraph of $\mathcal{D}(G)$ of type $A_{k}$ with $\gamma$ an extremal node corresponds to a linear space $\mathbb{P}^{k}$ in $G / P$. A subgraph of $\mathcal{D}(G)$ of type $A_{\ell}$ with $\gamma$ a node in the middle corresponds to a sub-Grassmannian. A Schubert variety of this type exists when $G$ is of type $A_{n}, D_{n}, E_{6}, E_{7}$.

(2) A subgraph of $\mathcal{D}(G)$ of type $D_{\ell}$ with $\gamma$ an extremal node corresponds to a quadric or an isotropic Grassmannian in $G / P$. A Schubert variety of this type exists when $G$ is of type $D_{n}, E_{6}, E_{7}$.

(3) A subgraph of $\mathcal{D}(G)$ of type $C_{\ell}$ with $\gamma=\alpha_{\ell}$ (in the usual notation of simple roots) corresponds to Lagrangian Grassmannian $L_{n}$ in $G / P$. A Schubert variety of this type exists when $G / P$ is Lagrangian Grassmannian $L_{m}$.

These are all smooth Schubert varieties in $G / P$ (Proposition 2.12).

\section{Criterions For the Rigidity}

3.1. Schubert rigidity. Schubert differential systems are $F$-structures for various $F$ 's, and the Schubert rigidity can be proved by showing the vanishing of a certain cohomology space.

Definition 3.1. Let $F$ be a submanifold of $G r(k, V)$ with a transitive action of a subgroup of $G L(V)$, where $\operatorname{dim} V=n$. A fiber bundle $\mathcal{F} \subset \operatorname{Gr}(k, T M)$ on a manifold $M$ of dimension $n$ is said to be an $F$-structure if at each point $x \in M$ there is a linear isomorphism $\varphi(x): V \rightarrow T_{x} M$ such that the induced map $\varphi(x)^{k}$ : $\operatorname{Gr}(k, V) \rightarrow \operatorname{Gr}\left(k, T_{x} M\right)$ sends $F$ to $\mathcal{F}_{x}$.

One can get the information on the integrability and on the set of integral varieties of an $F$-structure from certain cohomology spaces $H^{k, \ell}(F)$ depending only on the embedding $F \subset G r(k, V)$ (Chapter 1 of [G]).

The first cohomology $H^{1,1}(F)$ is defined by

$$
H^{1,1}(F)=\operatorname{Ker}\left(\partial: W_{f}^{*} \otimes T_{f} F \rightarrow \bigwedge^{2} W_{f}^{*} \otimes\left(V / W_{f}\right)\right),
$$

where $W_{f}$ stands for the $k$-subspace of $V$ represented by $f \in F$, and for $p: W_{f} \rightarrow$ $T_{f} F, \partial p: \bigwedge^{2} W_{f} \rightarrow V / W_{f}$ is defined by $\partial p\left(V_{1}, V_{2}\right)=p\left(V_{1}\right)\left(V_{2}\right)-p\left(V_{2}\right)\left(V_{1}\right)$, considering $T_{f} F$ as a subspace of $W_{f}^{*} \otimes\left(V / W_{f}\right)$. Define $H^{j, 1}(F)$ inductively so that the vanishing of $H^{1,1}(F)$ imply the vanishing of $H^{j, 1}(F)$ for all $j$ and that for a given integrable $F$-structure, if $H^{n, 1}(F)=0$, then the family of all integral varieties passing through a fixed point and tangent to a fixed tangent subspace has dimension $\sum_{j \leq n-1} \operatorname{dim} H^{j, 1}(F)$ (Chapter 1 of [G]). In short,

Proposition 3.2. Let $\mathcal{F}$ be an integrable $F$-structure. If $H^{1,1}(F)=0$, then for a fixed $x \in M$ and $y \in \mathcal{F}_{x}$, there exists a unique integral variety of $\mathcal{F}$ passing through $x$ and tangent to $W_{y}$.

Now we apply Proposition 3.2 to the Schubert differential system $\mathcal{B}_{w}$ on the Hermitian symmetric space $G / P$ as a $B_{w}$-structure. Since $P_{0}$ acts on the fiber $B_{w}$ transitively, we have the decomposition $\mathfrak{p}_{0}=\mathfrak{m}_{w}+\mathfrak{l}_{w}+\mathfrak{m}_{w}^{*}$, where $\mathfrak{l}_{w}+\mathfrak{m}_{w}^{*}$ is the Lie algebra of the isotropy group. Then the tangent space $T_{\left[\mathfrak{n}_{w]}\right]} B_{w} \subset T_{\left[\mathfrak{n}_{w]}\right]} G r(k, \mathfrak{m})$ is isomorphic to $\mathfrak{m}_{w} \subset \mathfrak{n}_{w}^{*} \otimes \mathfrak{m} / \mathfrak{n}_{w}$. 
Proposition 3.3. Let $G / P$ be a Hermitian symmetric space. Fix $w \in W^{P}$. Let $B_{w}$ be the fiber of the Schubert differential system $\mathcal{B}_{w}$. If

$$
H^{1,1}\left(B_{w}\right)=\operatorname{Ker}\left(\partial: \mathfrak{n}_{w}^{*} \otimes \mathfrak{m}_{w} \rightarrow \bigwedge^{2} \mathfrak{n}_{w}^{*} \otimes \mathfrak{m} / \mathfrak{n}_{w}\right)
$$

is zero, then $X_{w}$ is Schubert rigid.

3.2. The equality $B_{w}=R_{w}$. To find when $B_{w}$ is equal to $R_{w}$, we will compare their tangent spaces.

Note that under the embedding $G r(k, \mathfrak{m}) \subset \mathbb{P}\left(\bigwedge^{k} \mathfrak{m}\right)$, a tangent vector $\varphi$ in $T_{\left[\mathfrak{n}_{w]}\right]} G r(k, \mathfrak{m}) \simeq \mathfrak{n}_{w}^{*} \otimes \mathfrak{m} / \mathfrak{n}_{w}$ can be considered as a tangent vector $\varphi^{k}$ in

$$
T_{\left[\bigwedge^{k} \mathfrak{n}_{w}\right]} \mathbb{P}\left(\bigwedge^{k} \mathfrak{m}\right) \simeq \bigwedge^{k} \mathfrak{n}_{w}^{*} \otimes\left(\bigwedge^{k} \mathfrak{m} / \bigwedge^{k} \mathfrak{n}_{w}\right)
$$

in the following way. Take a basis $\left\{v_{1}, \cdots, v_{k}\right\}$ of $\mathfrak{n}_{w}$. For a linear map $\varphi: \mathfrak{n}_{w} \rightarrow$ $\mathfrak{m} / \mathfrak{n}_{w}$, the map $\varphi^{k}: \bigwedge^{k} \mathfrak{n}_{w} \rightarrow \bigwedge^{k} \mathfrak{m} / \bigwedge^{k} \mathfrak{n}_{w}$ is given by

$$
\varphi^{k}\left(v_{1} \wedge \cdots \wedge v_{k}\right)=\sum_{i} v_{1} \wedge \cdots \wedge \varphi\left(v_{i}\right) \wedge \cdots \wedge v_{k} \quad \bmod \bigwedge^{k} \mathfrak{n}_{w} .
$$

Proposition 3.4. Suppose that for the highest weight vector $\varphi$ of every irreducible $\mathfrak{l}_{w}$-representation space in the complement of $\mathfrak{m}_{w}$ in $\mathfrak{n}_{w}^{*} \otimes \mathfrak{m} / \mathfrak{n}_{w}$,

$$
\varphi^{k}\left(\bigwedge^{k} \mathfrak{n}_{w}\right) \not \subset \mathbf{I}_{w} / \bigwedge^{k} \mathfrak{n}_{w}
$$

Then $B_{w}$ is equal to $R_{w}$.

Proof. Put

$$
T_{w}=\left\{\varphi: \mathfrak{n}_{w} \rightarrow \mathfrak{m} / \mathfrak{n}_{w} \mid \varphi^{k}\left(\bigwedge^{k} \mathfrak{n}_{w}\right) \subset \mathbf{I}_{w} / \bigwedge^{k} \mathfrak{n}_{w}\right\}
$$

Since the space $T_{w}$ is equal to the intersection $T_{\left[\mathfrak{n}_{w]}\right.} G r(k, \mathfrak{m}) \cap T_{\left[\wedge^{k} \mathfrak{n}_{w]}\right.} \mathbb{P}\left(\mathbf{I}_{w}\right)$ and each space is invariant by the action of $\mathfrak{l}_{w}, T_{w}$ is an $\mathfrak{l}_{w}$-representation space. The tangent space $T_{\left[\mathfrak{n}_{w}\right]} B_{w}=\mathfrak{m}_{w}$ is contained in $T_{w}$. By the hypothesis, the complement of $\mathfrak{m}_{w}$ in $T_{\left[\mathfrak{n}_{w}\right]} G r(k, \mathfrak{m})$ intersects $T_{\left[\wedge^{k} \mathfrak{n}_{w}\right]} \mathbb{P}\left(\mathbf{I}_{w}\right)$ trivially. Thus the tangent space $T_{\left[\mathfrak{n}_{w}\right]} B_{w}=\mathfrak{m}_{w}$ is equal to $T_{w}$ in $\mathfrak{n}_{w}^{*} \otimes \mathfrak{m} / \mathfrak{n}_{w}$. Then since the tangent space $\mathfrak{m}_{w}$ of $B_{w}$ is contained in the tangent space $T_{\left[\wedge^{k} \mathfrak{n}_{w}\right]} R_{w}$ of $R_{w}$ and the latter is contained in $T_{w}, B_{w}$ and $R_{w}$ have the same tangent space at $\left[\mathfrak{n}_{w}\right]$. Hence $B_{w}$ is equal to $R_{w}$.

3.3. Lie algebra cohomology. The complement of $\mathfrak{m}_{w}$ in $\mathfrak{n}_{w}^{*} \otimes \mathfrak{m} / \mathfrak{n}_{w}$, together with $H^{1,1}\left(B_{w}\right)$, can be computed using Lie algebra cohomology as follows.

Proposition 3.5. For $w \in W^{P}$, define the action $\rho$ of $\mathfrak{n}_{w}$ on the space $\mathfrak{m}_{w}+\mathfrak{m} / \mathfrak{n}_{w}$ by

$$
\rho(A)(X)= \begin{cases}X(A) \in \mathfrak{m} / \mathfrak{n}_{w} & \text { for } X \in \mathfrak{m}_{w} \subset \mathfrak{n}_{w}^{*} \otimes\left(\mathfrak{m} / \mathfrak{n}_{w}\right), \\ 0 & \text { for } X \in \mathfrak{m} / \mathfrak{n}_{w}\end{cases}
$$

Then the Lie algebra cohomology $H^{1}\left(\mathfrak{n}_{w}, \mathfrak{m}_{w}+\mathfrak{m} / \mathfrak{n}_{w}\right)$ associated to the representation of the nilpotent Lie algebra $\mathfrak{n}_{w}$ on $\mathfrak{m}_{w}+\mathfrak{m} / \mathfrak{n}_{w}$ is the direct sum of $H^{1,1}\left(B_{w}\right)=$ $\operatorname{Ker}\left(\partial: \mathfrak{n}_{w}^{*} \otimes \mathfrak{m}_{w} \rightarrow \wedge^{2} \mathfrak{n}_{w}^{*} \otimes\left(\mathfrak{m} / \mathfrak{n}_{w}\right)\right)$ and the complement of $\mathfrak{m}_{w}$ in $\mathfrak{n}_{w}^{*} \otimes \mathfrak{m} / \mathfrak{n}_{w}$.

Proof. The complex defining the Lie algebra cohomology $H\left(\mathfrak{n}_{w}, \mathfrak{m}_{w}+\mathfrak{m} / \mathfrak{n}_{w}\right)$,

$$
\mathfrak{m}_{w}+\mathfrak{m} / \mathfrak{n}_{w} \rightarrow \mathfrak{n}_{w}^{*} \otimes\left(\mathfrak{m}_{w}+\mathfrak{m} / \mathfrak{n}_{w}\right) \rightarrow \wedge^{2} \mathfrak{n}_{w}^{*} \otimes\left(\mathfrak{m}_{w}+\mathfrak{m} / \mathfrak{n}_{w}\right) \rightarrow \quad \cdots,
$$


is the direct sum of the following subcomplexes:

$$
\begin{aligned}
& 0 \quad \rightarrow \quad \mathfrak{n}_{w}^{*} \otimes \mathfrak{m}_{w} \quad \rightarrow \quad \bigwedge^{2} \mathfrak{n}_{w}^{*} \otimes\left(\mathfrak{m} / \mathfrak{n}_{w}\right) \rightarrow \quad \cdots, \\
& \mathfrak{m}_{w} \rightarrow \mathfrak{n}_{w}^{*} \otimes\left(\mathfrak{m} / \mathfrak{n}_{w}\right) \quad \rightarrow \quad 0 \quad \rightarrow \quad \cdots .
\end{aligned}
$$

Since $\left[\mathfrak{n}_{w}, \mathfrak{n}_{w}\right]=0$, the boundary map of the first complex (1) is the same as the $\operatorname{map} \partial$.

In general, computing the Lie algebra cohomology $H^{1}\left(\mathfrak{n}_{w}, \mathfrak{m}_{w}+\mathfrak{m} / \mathfrak{n}_{w}\right)$ is not easy. However, if there is a semisimple Lie algebra $\mathfrak{q}_{0}$ which contains $\mathfrak{n}_{w}$, such that

(1) $\mathfrak{n}_{w}$ is the maximal nilpotent ideal of a parabolic subalgebra of $\mathfrak{q}_{0}$,

(2) the representation of $\mathfrak{n}_{w}$ on $\mathfrak{m}_{w}+\mathfrak{m} / \mathfrak{n}_{w}$ is the restriction of a representation of $\mathfrak{q}_{0}$ on $\mathfrak{m}_{w}+\mathfrak{m} / \mathfrak{n}_{w}$

then it can be calculated by the work of Kostant ([K1]). When the Schubert variety $X_{w}$ is associated with a subgraph of the Dynkin diagram $\mathcal{D}(G)$ of $G$, we can find such a Lie algebra $\mathfrak{q}_{0}$ in a natural way, which will be treated in the following section.

3.4. Subdiagrams of the marked Dynkin diagram $(\mathcal{D}(G), \gamma)$. Fix a connected subgraph $\delta$ of $(\mathcal{D}(G), \gamma)$ with $\gamma$ a node. Let $X_{w}$ be a smooth Schubert variety corresponding to $\delta$. In this case, the Schubert rigidity of $X_{w}$ can be checked by the same method as in $[\mathrm{CH}$, where they deal with the cases when the Schubert varieties are linear spaces after $G / P$ is embedded in the projective space minimally. In the following we collect the facts that are needed to deal with the cases studied in this paper.

From now on, to avoid negative signs in the computation, we will adopt the convention that the nilpotent part of Borel or parabolic subalgebras are generated by root spaces of negative roots. Then the tangent space of the homogeneous space $G / P$ is generated by root spaces of positive roots.

For $\alpha \in \mathcal{S}$, let $\sigma_{\alpha}$ denote the reflection with respect to $\alpha$, which is an element of the Weyl group of $\mathfrak{g}$. For a nilpotent Lie algebra $\mathfrak{n}$ of $\mathfrak{g}$, let $\Delta(\mathfrak{n})$ denote the set of all roots $\beta$ with $\mathfrak{g}_{\alpha} \subset \mathfrak{n}$. Then $\mathfrak{n}$ can be written as $\mathfrak{n}=\sum_{\alpha \in \Delta(\mathfrak{n})} \mathfrak{g}_{\alpha}$.

For $\alpha_{i} \in \mathcal{S}$, define $n_{\alpha_{i}}: \Delta \rightarrow \mathbb{Z}$ by $n_{\alpha_{i}}(\alpha)=n_{i}$ for $\alpha=\sum_{j} n_{j} \alpha_{j}$. For a subset $\mathcal{S}_{1}=\left\{\alpha_{i_{1}}, \cdots, \alpha_{i_{r}}\right\}$ of $\mathcal{S}$, define the map $n_{\mathcal{S}_{1}}$ by

$$
\begin{aligned}
& n_{\mathcal{S}_{1}}: \Delta^{+} \longrightarrow \mathbb{Z}^{|I|}, \\
& \alpha=\sum n_{i} \alpha_{i} \longmapsto\left(n_{i_{1}}, \cdots, n_{i_{r}}\right) .
\end{aligned}
$$

For a connected subgraph $\delta$ of $\mathcal{D}(G)$, define the neighborhood $N(\delta)$ of $\delta$ by the set of simple roots that are not in $\delta$ but that are connected to $\delta$ by an edge.

Consider the map $n_{\mathcal{S}_{1}}$ for $\mathcal{S}_{1}=N(\delta)$. For each $\mathbf{i}=\left(n_{i_{1}}, \cdots, n_{i_{r}}\right)$ in the image $n_{N(\delta)}\left(\Delta^{+}\right) \subset \mathbb{Z}^{|N(\delta)|}$, let $\lambda_{\mathbf{i}}$ denote the root in $\left\{\alpha \in \Delta^{+}: n_{N(\delta)}(\alpha)=\mathbf{i}\right\}$ of maximal height. Let $D$ be the set of all such $\lambda_{\mathbf{i}}$.

Proposition 3.6. Let $\delta$ be a connected subgraph of $(\mathcal{D}(G), \gamma)$ with $\gamma$ a node. Let $X_{w}$ be a Schubert variety of type $w$ corresponding to $\delta$. Define a reductive Lie algebra $\mathfrak{q}_{0}$ by $\mathfrak{q}_{0}=\mathfrak{h}+\sum_{\alpha \in \Delta_{\delta}} \mathfrak{g}_{\alpha}$, where $\Delta_{\delta}=\Delta_{\mathcal{S} \backslash N(\delta)}$, and define $D$ as above. Then $H^{1}\left(\mathfrak{n}_{w}, \mathfrak{m}_{w}+\mathfrak{m} / \mathfrak{n}_{w}\right)$ is the direct sum of irreducible $\mathfrak{q}_{0}$-representation spaces $H^{1}\left(\mathfrak{n}_{w}, \mathfrak{m}_{w}+\mathfrak{m} / \mathfrak{n}_{w}\right)_{\lambda}, \lambda \in D$. The highest weight vector of $H^{1}\left(\mathfrak{n}_{w}, \mathfrak{m}_{w}+\mathfrak{m} / \mathfrak{n}_{w}\right)_{\lambda}$ is $x_{\gamma}^{*} \otimes x_{\sigma_{\gamma}(\lambda)}$. 
Proof. Set $\mathfrak{n}=\sum_{\alpha \in \Delta+\backslash \Delta_{\delta}} \mathfrak{g}_{\alpha}$. Then $\mathfrak{q}_{0}+\mathfrak{n}^{*}$ is the parabolic subalgebra generated by $\mathcal{S} \backslash N(\delta)$ and we have $\mathfrak{g}=\mathfrak{n}+\mathfrak{q}_{0}+\mathfrak{n}^{*}$. The nilpotent Lie algebras $\mathfrak{n}, \mathfrak{m}, \mathfrak{n}_{w}, \mathfrak{m}_{w}$ are generated by the root spaces of roots in

$$
\begin{aligned}
\Delta(\mathfrak{n}) & =\left\{\alpha \in \Delta^{+}: n_{\beta}(\alpha)>0 \text { for some } \beta \in N(\delta)\right\}, \\
\Delta(\mathfrak{m}) & =\left\{\alpha \in \Delta^{+}: n_{\gamma}(\alpha)=1\right\}, \\
\Delta\left(\mathfrak{m}_{w}\right) & =\left\{\alpha \in \Delta^{+}: n_{\gamma}(\alpha)=0, n_{\beta}(\alpha)>0 \text { for some } \beta \in N(\delta)\right\}, \\
\Delta\left(\mathfrak{n}_{w}\right) & =\left\{\alpha \in \Delta^{+}: n_{\gamma}(\alpha)=1, n_{\beta}(\alpha)=0\right\} .
\end{aligned}
$$

So we have $\mathfrak{n}=\mathfrak{m}_{w}+\mathfrak{m} / \mathfrak{n}_{w}$ and $\mathfrak{q}_{0}=\mathfrak{n}_{w}+\mathfrak{l}_{w}+\mathfrak{n}_{w}^{*}$. Furthermore, the restriction of the adjoint action of $\mathfrak{q}_{0}$ on $\mathfrak{n}$ to $\mathfrak{n}_{w}$ is equal to the action of $\mathfrak{n}_{w}$ on $\mathfrak{m}_{w}+\mathfrak{m} / \mathfrak{n}_{w}$ defined in Proposition 3.5 .

For each $\mathbf{i}=\left(n_{i_{1}}, \cdots, n_{i_{r}}\right)$ in the image $n_{N(\delta)}\left(\Delta^{+}\right) \subset \mathbb{Z}^{|N(\delta)|}$, let $\mathfrak{n}_{\mathbf{i}}$ denote the nilpotent Lie subalgebra spanned by the root vectors $x_{\alpha}$ of root $\alpha$ with $n_{N(\delta)}(\alpha)=\mathbf{i}$. Then $\mathfrak{n}$ is decomposed as $\sum_{\mathbf{i}} \mathfrak{n}_{\mathbf{i}}$ and each $\mathfrak{n}_{\mathbf{i}}$ is an irreducible $\mathfrak{q}_{0}$-representation space with highest weight $\lambda_{\mathbf{i}}$ (Section 9.9 of $[\mathrm{BE}]$ ). Since the action by $\mathfrak{n}_{w}$ does not change the coefficient $n_{\beta}(\alpha)$ for $\beta \in N(\delta), H^{1}\left(\mathfrak{n}_{w}, \mathfrak{n}\right)$ is the direct sum of $H^{1}\left(\mathfrak{n}_{w}, \mathfrak{n}_{\mathbf{i}}\right)$ 's. By Theorem 5.14 of [K1], each $H^{1}\left(\mathfrak{n}_{w}, \mathfrak{n}_{\mathbf{i}}\right)$ is an irreducible representation space of $\mathfrak{l}_{w}$ with the highest weight vector $x_{\gamma}^{*} \otimes x_{\sigma_{\gamma}\left(\lambda_{\mathbf{i}}\right)}$.

Proposition 3.7. With the same notations as in Proposition 3.6, put

$$
\begin{aligned}
D^{\prime} & =\left\{\lambda \in D: n_{\gamma}\left(\sigma_{\gamma}(\lambda)\right)=0\right\} \\
& =\left\{\lambda \in D: \sum_{\beta \in N(\gamma)} n_{\beta}(\lambda)=n_{\gamma}(\lambda)\right\} .
\end{aligned}
$$

Then $H^{1,1}\left(B_{w}\right)$ is the direct summand of $H^{1}\left(\mathfrak{n}_{w}, \mathfrak{m}_{w}+\mathfrak{m} / \mathfrak{n}_{w}\right)$ consisting of $\mathfrak{l}_{w}$ modules with the highest weight vector $x_{\gamma}^{*} \otimes x_{\sigma_{\gamma}(\lambda)}$ for $\lambda \in D^{\prime}$. Therefore, $X_{w}$ is Schubert rigid if $D^{\prime}=\emptyset$.

Proof. It suffices to show that $n_{\gamma}\left(\sigma_{\gamma}(\lambda)\right)=0$ if and only if $\sum_{\beta \in N(\gamma)} n_{\beta}(\lambda)-n_{\gamma}(\lambda)=$ 0. By definition, $\sigma_{\gamma}(\alpha)=\alpha-\left\langle\alpha, \gamma^{\vee}\right\rangle \gamma$. For a simple root $\alpha$,

$$
\left\langle\alpha, \gamma^{\vee}\right\rangle=\left\{\begin{array}{l}
2 \text { if } \alpha=\gamma, \\
-1 \text { if } \alpha \in N(\gamma), \\
0 \text { otherwise, }
\end{array}\right.
$$

so we have $n_{\gamma}\left(\sigma_{\gamma}(\lambda)\right)=\sum_{\beta \in N(\gamma)} n_{\beta}(\lambda)-n_{\gamma}(\lambda)$. This gives the desired equality.

Proposition 3.8. With the same notations as in Proposition 3.6, put

$$
\begin{aligned}
D^{\prime \prime} & =\left\{\lambda \in D: n_{\gamma}\left(\sigma_{\gamma}(\lambda)\right)=1\right\} \\
& =\left\{\lambda \in D: \sum_{\beta \in N(\gamma)} n_{\beta}(\lambda)=n_{\gamma}(\lambda)+1\right\} .
\end{aligned}
$$

Take a basis $\left\{v_{1}, \cdots, v_{k}\right\}$ of $\mathfrak{n}_{w}$ consisting of weight vectors and with $v_{1}=x_{\gamma}$. If $x_{\sigma_{\gamma}(\lambda)} \wedge v_{2} \wedge \cdots \wedge v_{k}$ is not contained in $\mathbf{I}_{w}$ for any $\lambda \in D^{\prime \prime}$, then $B_{w}$ is equal to $R_{w}$

Proof. By Proposition 3.4 and Proposition 3.5. to show that $\mathfrak{m}_{w}$ is equal to $T_{w}$, it suffices to check for these generators $x_{\gamma}^{*} \otimes x_{\sigma_{\gamma}(\lambda)}$ with $\lambda \in D^{\prime \prime}$ that the image of the map $\varphi_{\lambda}^{k}$ induced by $\varphi_{\lambda}=x_{\gamma}^{*} \otimes x_{\sigma_{\gamma}(\lambda)}$ is not contained in $\mathbf{I}_{w}$. Since we take $v_{1}=x_{\gamma}$, $\varphi_{\lambda}^{k}\left(v_{1} \wedge \cdots \wedge v_{k}\right)=x_{\sigma_{\gamma}(\lambda)} \wedge v_{2} \cdots \wedge v_{k}$. 
The Schubert differential system $\mathcal{B}_{w}$ and the Schubert rigidity can be defined in general homogeneous space $G / P$ in the same way as in the case of Hermitian symmetric spaces. Suppose that $P$ is a maximal parabolic corresponding to a simple root $\gamma$. To a subdiagram $\delta$ of $\mathcal{D}(G)$ with $\gamma$ a node, associate a Schubert variety $X_{w}$ in $G / P$ in the same way. Assume that the marked diagram $(\delta, \gamma)$ represents a Hermitian symmetric space. Then the tangent space $\mathfrak{n}_{w}$ of $X_{w}$ satisfies $\left[\mathfrak{n}_{w}, \mathfrak{n}_{w}\right]=0$. So we can apply the same method as above to check the Schubert rigidity of such a Schubert variety. Note that $\mathfrak{m}$ is not irreducible: $\mathfrak{m}=\sum \mathfrak{m}_{i}$, where $\mathfrak{m}_{i}=\sum_{n_{\gamma}(\alpha)=i} \mathfrak{g}_{\alpha}$. However, $\mathfrak{n}_{w}$ is contained in $\mathfrak{m}_{1}$, and the complex defining Lie algebra cohomology $H\left(\mathfrak{n}_{w}, \mathfrak{m}_{w}+\mathfrak{m} / \mathfrak{n}_{w}\right)$ is decomposed as follows:

$$
\begin{aligned}
& 0 \quad \rightarrow \quad \mathfrak{n}_{w}^{*} \otimes \mathfrak{m}_{w} \quad \rightarrow \quad \bigwedge^{2} \mathfrak{n}_{w}^{*} \otimes\left(\mathfrak{m}_{1} / \mathfrak{n}_{w}\right) \quad \rightarrow \quad \ldots \\
& \mathfrak{m}_{w} \rightarrow \mathfrak{n}_{w}^{*} \otimes\left(\mathfrak{m}_{1} / \mathfrak{n}_{w}\right) \rightarrow \bigwedge^{2} \mathfrak{n}_{w}^{*} \otimes \mathfrak{m}_{2} \quad \rightarrow \quad \ldots \\
& \mathfrak{m}_{1} \rightarrow \quad \mathfrak{n}_{w}^{*} \otimes \mathfrak{m}_{2} \quad \rightarrow \quad \bigwedge^{2} \mathfrak{n}_{w}^{*} \otimes \mathfrak{m}_{3} \quad \rightarrow \quad \ldots
\end{aligned}
$$

$\cdots$

Thus $X_{w}$ is Schubert rigid if $D^{\prime}=\emptyset$ as in Proposition 3.7 .

\section{Schubert Rigidity AND Schur Rigidity}

4.1. Schubert rigidity. Let $\delta_{0}$ be the subgraph of $\mathcal{D}(G)$ of type $A_{\ell}$ with two ends, $\gamma$ and the branch point (the node connected to three other points). Let $k_{G / P}$ be the length of $\delta_{0}$. Then for any $j \leq k_{G / P}, H^{2 j}(G / P, \mathbb{Z}) \simeq \mathbb{Z}$, and thus the homology class of any subvariety of dimension $j \leq k_{G / P}$ is a multiple of the homology class of the Schubert variety $\mathbb{P}^{j}, j \leq k_{G / P}$, in $G / P$. So these Schubert varieties are not Schur rigid.

Proposition 4.1. Let $G / P$ be a Hermitian symmetric space which is not an odd-dimensional quadric. Let $(\delta, \gamma)$ be a subgraph of $(\mathcal{D}(G), \gamma)$ of type $\left(A_{k}, \alpha_{1}\right)$ with length $>k_{G / P}$ and let $\delta_{\max }$ be the unique maximal one among subgraphs of $(\mathcal{D}(G), \gamma)$ of type $\left(A_{n}, \alpha_{1}\right)$ containing $\delta$.

Let $X_{w}$ be a Schubert variety corresponding to $\delta$. Then integral varieties of $\mathcal{B}_{w}$ are subvarieties of a Schubert variety of type corresponding to $\delta_{\max }$. Therefore $X_{w}$ is Schubert rigid if $X_{w}$ is a maximal linear space.

Proof. See [CH].

Lemma 4.2. Let $(\mathcal{D}(G), \gamma)$ be a marked Dynkin diagram and let $(\delta, \gamma)$ be a subgraph. For $\mathbf{i} \in n_{N(\delta)}\left(\Delta^{+}\right)$, let $\lambda_{\mathbf{i}}$ be the root of maximal height among $\alpha \in \Delta^{+}$with $n_{N(\delta)}(\alpha)=\mathbf{i}$. If $(\delta, \gamma)$ is not of type $\left(A_{k}, \alpha_{1}\right)$, then $n_{\gamma}\left(\sigma_{\gamma}\left(\lambda_{\mathbf{i}}\right)\right)>0$ for all $\mathbf{i}$.

Proof. From the proof of Proposition 3.7 it follows that

$$
n_{\gamma}\left(\sigma_{\gamma}\left(\lambda_{\mathbf{i}}\right)\right) \geq \sum_{\beta \in N(\gamma)} n_{\beta}\left(\lambda_{\mathbf{i}}\right)-n_{\gamma}\left(\lambda_{\mathbf{i}}\right) .
$$

If $(\delta, \gamma)$ is not of type $\left(A_{k}, \alpha_{1}\right)$, then we have $\sum_{\beta \in N(\gamma)} n_{\beta}\left(\lambda_{\mathbf{i}}\right)>n_{\gamma}\left(\lambda_{\mathbf{i}}\right)$ for every $\mathbf{i} \in$ $n_{N(\delta)}\left(\Delta^{+}\right)$because $\lambda_{\mathbf{i}}$ has maximal height among $\alpha \in \Delta^{+}$with $n_{N(\delta)}(\alpha)=\mathbf{i}$.

Proposition 4.3. Let $G / P$ be a Hermitian symmetric space. Let $(\delta, \gamma)$ be a subgraph of $(\mathcal{D}(G), \gamma)$ which is not of type $\left(A_{k}, \alpha_{1}\right)$. Then the corresponding Schubert variety $X_{w}$ of $G / P$ is Schubert rigid. 
Proof. Use Proposition 3.7 and Lemma 4.2 .

Lemma 4.2 holds for general homogeneous space. By the remark at the end of Section 3.4, we have

Proposition 4.4. Let $G / P$ be a homogeneous space with $P \subset G$ a maximal parabolic subgroup corresponding to $\gamma$. Let $\delta$ be a subgraph of $\mathcal{D}(G)$ with $\gamma$ a node. Suppose that the marked diagram $(\delta, \gamma)$ represents a Hermitian symmetric space but that it is not of type $\left(A_{k}, \alpha_{1}\right)$. Then the corresponding Schubert variety $X_{w}$ in $G / P$ is Schubert rigid.

Example 4.5. Schubert varieties as in Proposition 4.4 arise when we consider the desingularization of singular Schubert varieties in Hermitian symmetric space.

Let $G / P$ be an even-dimensional quadric $Q^{2 n} \subset \mathbb{P}(V)$ and let $X_{w}$ be a singular Schubert variety of codimension 1 . Then $X_{w}$ may be thought of as the locus of the family of $\mathbb{P}^{1}$ 's in $Q^{2 n}$ passing through a fixed point $p \in Q$, or, equivalently, of the family of $\mathbb{P}(E)$ 's for isotropic 2-subspaces $E$ of $V$ containing a fixed isotropic 1-subspace $W$.

For an isotropic 2-subspace $E$ of $V, \mathbb{P}(E)$ is a smooth Schubert variety in $Q^{2 n}$, say, of type $v$. The family of isotropic 2-subspaces of $V$ is a homogeneous space, say, $G / Q$. Then the subvariety of $G / Q$ consisting of isotropic 2-subspaces containing a fixed isotropic 1-subspace $W$ is a smooth Schubert variety $X_{u}$ of $G / Q$ of the form as in Proposition 4.4. Thus we can write $X_{w}=\bigcup_{W \subset E} \mathbb{P}(E)$ as the locus $\bigcup_{t \in X_{u}}\left(X_{v}\right)_{t}$ of the family $\left\{\left(X_{v}\right)_{t}\right\}$ of smooth Schubert varieties of type $v$ in $G / P$, parameterized by a smooth Schubert variety $X_{u}$ of type $u$ in $G / Q$.

In general, a singular Schubert variety $X_{w}$ in $Q^{2 n}$ of codimension $r<n-1$ can be expressed as the locus of the family of smooth Schubert varieties in $Q^{2 n}$ parameterized by a smooth Schubert variety in $G / Q$ of the form as in Proposition 4.4.

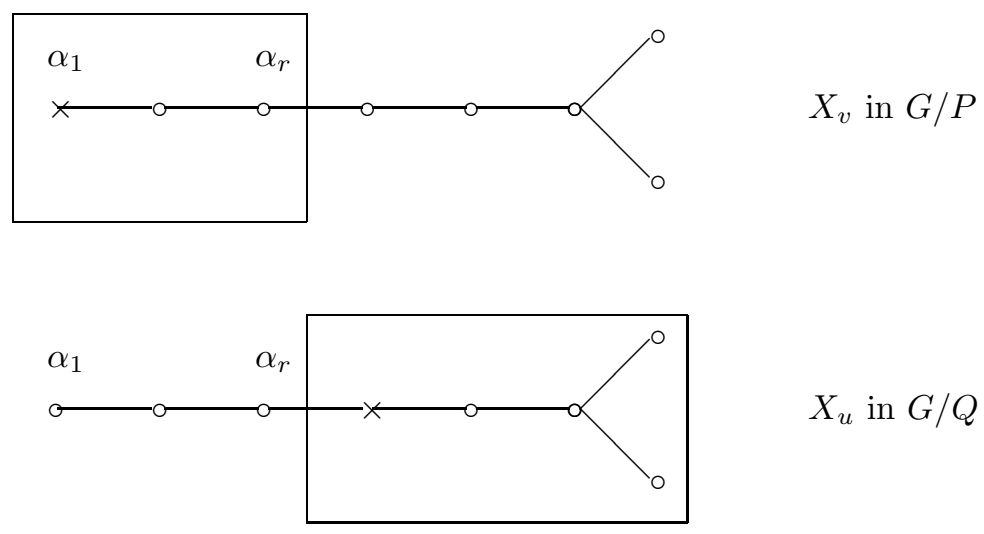

$X_{w}$, a singular Schubert variety of codimension $r$ in $G / P=Q^{2 n}$ 
A desingularization of $X_{w}$ can be obtained by considering the following double fibration:

$$
G r_{i s o}(r+1, V)=G / Q \stackrel{\mu}{\longleftarrow} \operatorname{Flag}_{i s o}(1, r+1, V) \stackrel{\nu}{\longrightarrow} Q^{2 n}=G / P .
$$

Fix an isotropic $r$-subspace $W$ in $V$. Then the union of $\mathbb{P}^{r}$ 's in $Q^{2 n}$ containing $\mathbb{P}(W)$ is a Schubert variety $X_{w}$ of type $w$ in $Q^{2 n}=G / P$ of codimension $r$. The subvariety consisting of isotropic $(r+1)$-subspaces of $V$ containing $W$ is a Schubert variety $X_{u}$ of type $u$ in $G r_{i s o}(r+1, V)=G / Q$. The preimage $\mu^{-1}\left(X_{u}\right) \subset$ Flagiso $_{i s}(1, r+1, V)$ is smooth and maps to $X_{w}$ birationally by $\nu$.

4.2. The equality $B_{w}=R_{w}: G r(m, n)$-case. First, we review how to construct the Schur module (or Weyl module) $\mathbb{S}_{\mathbf{a}}(V)$ for a vector space $V$ of dimension $n$ and for a partition $\mathbf{a}=\left(a_{1}, \cdots, a_{n}\right)$. For references, see Chapter 6 and Chapter 15 of [FH] and Chapter 8 of $[\mathrm{F}$.

Let $\mathbf{a}^{\prime}$ be the conjugate of $\mathbf{a}$ and $k=|\mathbf{a}|$. Put the factors of the $k$-th tensor product $V^{\otimes k}$ in one-to-one correspondence with the squares of the Young diagram of $\mathbf{a}$. Then $\mathbb{S}_{\mathbf{a}}(V)$ is the image of this composite map:

$$
\bigotimes_{i}\left(\bigwedge^{a_{i}^{\prime}} V\right) \rightarrow \bigotimes_{i}\left(\bigotimes^{a_{i}^{\prime}} V\right) \rightarrow V^{\otimes k} \rightarrow \bigotimes_{j}\left(\bigotimes^{a_{j}} V\right) \rightarrow \bigotimes_{j}\left(S y m^{a_{j}}(V)\right),
$$

the first map being the tensor product of the obvious inclusion, the second grouping the factors of $V^{\otimes k}$ according to the columns of the Young diagram, the third grouping the factors according to the rows of the Young diagram, and the fourth being the obvious quotient map (Exercise 6.14 of $[\mathrm{FH}]$ ).

Let $\left\{v_{1}, \cdots, v_{n}\right\}$ be a basis of $V$. For a semi-stable tableau $T$ of shape a (a numbering of the Young diagram of $\mathbf{a}$ in such a way that the number is strictly increasing in column and is nondecreasing in row), let $e_{T} \in \bigotimes_{i}\left(\bigwedge^{a_{i}^{\prime}} V\right)$ be a tensor product of wedge products of basis element for $V$, the $i$-th factor in $\bigwedge^{a_{i}^{\prime}} V$ being the wedge product (in order) of those basis vectors whose indices occur in the $i$ th column of $T$. Then the set of the images of these elements $e_{T}$ for semi-stable tableau $T$ is a basis of $\mathbb{S}_{\mathbf{a}}(V)$ (Theorem 1 of Section 8.1 of $[\mathrm{F}]$ ). Furthermore, under the action of $S L(V)$, each $e_{T}$ is a weight vector of weight $\mathbf{b}=\left(b_{1}, \cdots, b_{m}\right)$, where $b_{i}$ is the number of times the integer $i$ occurs in $T$ (Section 8.2 of $[\mathrm{F}]$ ). The highest weight vector corresponds to the semi-standard tableau $T$ with all the elements in $i$-th row equal to $i$, e.g., in $\mathbb{S}_{\mathbf{a}}(V)$ for $\mathbf{a}=(4,4,4,4)$,

\begin{tabular}{|l|l|l|l|}
\hline 1 & 1 & 1 & 1 \\
\hline 2 & 2 & 2 & 2 \\
\hline 3 & 3 & 3 & 3 \\
\hline
\end{tabular} corresponds to the weight vector of highest weight $(4,4,4,4)$ and \begin{tabular}{|l|l|l|l|}
\hline 1 & 2 & 2 & 3 \\
\hline 2 & 3 & 3 & 4 \\
\hline 3 & 4 & 5 & 5 \\
\hline
\end{tabular} corresponds to the weight vector of weight $(1,3,4,2,2)$.

The dimension of the weight space with weight $\mathbf{b}$ in the representation space $\mathbb{S}_{\mathbf{a}}\left(\mathbb{C}^{n}\right)$ is the number of ways one can fill the Young diagram of a with $b_{1} 1$ 's, $b_{2}$ 2 's, $\cdots b_{n} n$ 's, in such a way that the entries in each row are nondecreasing and 
those in each column are strictly increasing, e.g., for $\mathbf{a}=(4,4,4), \mathbf{b}=(4,3,2,2,1)$ is a weight in the representation space $\mathbb{S}_{\mathbf{a}}(V)$, but $\mathbf{b}=(5,4,3)$ is not.

Proposition 4.6. For $\mathbf{a}=\left(p^{q}\right)$ with $(p, q) \neq(1,1)$, the Schubert differential system $\mathcal{B}_{\mathbf{a}^{*}}$ is equal to the Schur differential system $\mathcal{R}_{\mathbf{a}}$.

We remark that the case when $p=1$ or $q=1$ was proved in Lemma 4 and Lemma 5 of $\underline{B}$.

Proof. In this case $\sigma_{\mathbf{a}^{*}}$ is $\left\{E \in G r(m, n): \mathbb{C}^{m-q} \subset E \subset \mathbb{C}^{m+p}\right\} \simeq G r(q, p+q)$. For simplicity, assume that $1<p<n-m$ and $1<q<m$ (other cases can be proved in a similar way). Let $\delta$ be the subdiagram consisting of the simple roots $\alpha_{i}$ for $m-q+1 \leq i \leq m+p-1$. Then $N(\delta)=\left\{\alpha_{m-q}, \alpha_{m+p}\right\}$ and $D=\left\{\lambda_{1,0}, \lambda_{0,1}, \lambda_{1,1}\right\}=$ $D^{\prime \prime}$, where $\lambda_{1,0}=\sum_{1 \leq i \leq m+p-1} \alpha_{i}, \lambda_{0,1}=\sum_{m-q+1 \leq i \leq n} \alpha_{i}$, and $\lambda_{1,1}=\sum_{1 \leq i \leq n} \alpha_{i}$. For all $\lambda \in D^{\prime \prime}$, we have $\sigma_{\gamma}(\lambda)=\lambda$.

Recall that $\mathbf{I}_{\mathbf{a}}$ is given by $\mathbb{S}_{\mathbf{a}}\left(E^{*}\right) \otimes \mathbb{S}_{\mathbf{a}^{\prime}}(Q)$. Choose a basis $\left\{e_{1}, \cdots, e_{m}\right\}$ (respectively, $\left\{q_{1}, \cdots, q_{n-m}\right\}$ ) of $E$ (respectively, of $Q$ ) such that the vector $e_{i}^{*} \otimes q_{j}$ is a root vector of root $\sum_{m-i+1 \leq s \leq m+j-1} \alpha_{s}$ in $\mathfrak{m}=E^{*} \otimes Q$. The basis of $\mathbb{S}_{\mathbf{a}}\left(E^{*}\right) \otimes \mathbb{S}_{\mathbf{a}^{\prime}}(Q)$ consisting of weight vectors is

\section{$\left\{e_{T}^{*} \otimes q_{S}: \quad T\right.$ is a semi-standard tableau of shape $\mathbf{a}$ and $S$ is a semi-standard tableau of shape $\left.\mathbf{a}^{\prime}\right\}$,}

where $e_{T}$ and $q_{S}$ are defined at the beginning of this section.

The vector space $\mathfrak{n}_{\mathbf{a}^{*}}$ is spanned by the vectors $e_{i}^{*} \otimes q_{j}, 1 \leq i \leq q, 1 \leq j \leq p$, and $\bigwedge^{k} \mathfrak{n}_{\mathbf{a}^{*}}=\bigwedge_{i, j}\left(e_{i}^{*} \otimes q_{j}\right)$ is a scalar multiple of $e_{T}^{*} \otimes q_{S}$ for the semi-standard tableau

$$
T=\begin{array}{|c|c|c|c|}
\hline 1 & 1 & \cdots & 1 \\
\hline 2 & 2 & \cdots & 2 \\
\hline \vdots & \vdots & & \vdots \\
\hline q & q & \cdots & q \\
\hline
\end{array} \quad \text { and } \quad S^{t}=\begin{array}{|c|c|c|c|}
\hline 1 & 2 & \cdots & p \\
\hline 1 & 2 & \cdots & p \\
\hline \vdots & \vdots & & \vdots \\
\hline 1 & 2 & \cdots & p \\
\hline
\end{array}
$$

Here, we denote by $S^{t}$ the transpose of $S$, by noting that the Young diagram of $\mathbf{a}^{\prime}$ is the transpose of the Young diagram of $\mathbf{a}$. This will help to understand the element $e_{T}^{*} \otimes q_{S}$ in the tensor product $\mathbb{S}_{\mathbf{a}}\left(E^{*}\right) \otimes \mathbb{S}_{\mathbf{a}^{\prime}}(Q)$. Also, note that $e_{T}^{*} \otimes q_{S}$ is not a decomposable $k$-vector in $\Lambda^{k}\left(E^{*} \otimes Q\right)$ for general $T$ and $S$, but that, for $T$ and $S$ as above, $e_{T}^{*} \otimes q_{S}$ is a highest weight vector, and it is a decomposable $k$-vector $\bigwedge_{i, j}\left(e_{i}^{*} \otimes q_{j}\right)$.

The weight vector $x_{\lambda_{1,0}}$ of weight $\lambda_{1,0}$ is $e_{m}^{*} \otimes q_{p}$. If we replace $e_{1}^{*} \otimes q_{1}$ by $e_{m}^{*} \otimes q_{p}$, then $\varphi_{1,0}^{k}:=\left(e_{m}^{*} \otimes q_{p}\right) \wedge\left(e_{1}^{*} \otimes q_{2}\right) \wedge \cdots \wedge\left(e_{q}^{*} \otimes q_{p}\right)$ is a weight vector of weight $(p-1, p, \cdots, p, 1) \oplus(q-1, q, \cdots, q, q+1)$. But $(q-1, q, \cdots, q, q+1)$ is not a weight of $\mathbb{S}_{\mathbf{a}^{\prime}}(Q)$. Thus $\varphi_{1,0}^{k}$ is not contained in $\mathbf{I}_{\mathbf{a}}=\mathbb{S}_{\mathbf{a}}\left(E^{*}\right) \otimes \mathbb{S}_{\mathbf{a}^{\prime}}(Q)$. Similarly, $\varphi_{0,1}^{k}=x_{\lambda_{0,1}} \wedge\left(e_{1}^{*} \otimes q_{2}\right) \wedge \cdots \wedge\left(e_{q}^{*} \otimes q_{p}\right)$ is not contained in $\mathbf{I}_{\mathbf{a}}$.

For $\lambda_{1,1}$, consider $\varphi_{1,1}^{k}=x_{\lambda_{1,1}} \wedge\left(e_{1}^{*} \otimes q_{2}\right) \wedge \cdots \wedge\left(e_{q}^{*} \otimes q_{p}\right)$, where $x_{\lambda_{1,1}}=e_{m}^{*} \otimes q_{n-m}$ is the weight vector of weight $\lambda_{1,1}$. Since $a d\left(x_{-\alpha_{m+p}-\cdots-\alpha_{n}}\right)$ does not change any vector in $\varphi_{1,1}^{k}$ except $x_{\lambda_{1,1}}$, we get

$$
\operatorname{ad}\left(x_{-\alpha_{m+p}-\cdots-\alpha_{n}}\right) \varphi_{1,1}^{k}=x_{\lambda_{1,0}} \wedge\left(e_{1}^{*} \otimes q_{2}\right) \wedge \cdots \wedge\left(e_{q}^{*} \otimes q_{p}\right) \notin \mathbf{I}_{\mathbf{a}} .
$$

Since $\operatorname{ad}\left(x_{-\alpha_{m+p}-\cdots-\alpha_{n}}\right)$ preserves each irreducible $\mathfrak{p}_{0}$-representation in $\bigwedge^{k} \mathfrak{m}, \varphi_{1,1}^{k}$ is not contained in $\mathbf{I}_{\mathbf{a}}$. By Proposition $3.8, B_{\mathbf{a}^{*}}$ is equal to $R_{\mathbf{a}}$. 
4.3. The equality $B_{w}=R_{w}$ : Hermitian symmetric space $\neq G r(m, n)$ case. To prove the equality $B_{w}=R_{w}$ for the smooth Schubert variety $X_{w}$ in Hermitian symmetric space $G / P \neq G r(m, n)$, we will consider a natural irreducible representation space $\mathbf{I}_{\tau}$ in $\bigwedge^{k} \mathfrak{m}$ other than $\mathbf{I}_{w}$ so that for any $\lambda \in D^{\prime \prime}, \varphi_{\lambda}^{k}=x_{\sigma_{\gamma}(\lambda)} \wedge v_{2} \wedge \cdots \wedge v_{k}$ has a non-zero $\mathbf{I}_{\tau}$-component, i.e., if we write $\varphi_{\lambda}^{k}=\sum_{\sigma} \varphi_{\sigma}$, where $\varphi_{\sigma} \in \mathbf{I}_{\sigma}$ according to the decomposition $\bigwedge^{k} \mathfrak{m}=\sum_{\sigma} \mathbf{I}_{\sigma}$, then $\varphi_{\tau} \neq 0$. So it will not be contained in $\mathbf{I}_{w}$. This will prove the equality $B_{w}=R_{w}$ by Proposition 3.8 .

Note that if $w=\sigma_{\beta_{1}} \cdots \sigma_{\beta_{k}}, \beta_{i} \in \mathcal{S}$, is a reduced expression of $w$, then

$$
\sigma_{\beta_{1}} \cdots \sigma_{\beta_{i-1}}\left(\beta_{i}\right), \quad i=1, \cdots, k,
$$

are all distinct and let $\Delta(w)$ consists of such roots.

Proposition 4.7. Let $G / P$ be a Hermitian symmetric space $\neq G r(m, n)$. Let $\delta$ be a subgraph of $\mathcal{D}(G)$ of type $A_{\ell}, \ell>k_{G / P}$, with $\gamma$ a node and let $X_{w}$ be the corresponding Schubert variety. Then $\mathcal{B}_{w}$ is equal to $\mathcal{R}_{w}$.

Here, $k_{G / P}$ is the length of the chain connecting $\gamma$ and the branch point as in Section 4.1

We remark that the case of the maximal linear spaces in the quadric $Q^{2 n}$ and the case of linear spaces of dimension $\geq 3$ in the isotropic Grassmannian $N_{2 n}^{+}$was proved in Lemma 12 and Lemma 13 of $[\mathrm{B}$.

Proof. First, we will prove the case of quadric $Q^{2 n}$ and then show that other cases can be reduced to the quadric case.

Special case. Assume that $G / P$ is $Q^{2(n-1)}$. Let $\mathcal{S}=\left\{\alpha_{1}, \cdots, \alpha_{n}\right\}$ be the set of simple roots indexed in the following way:

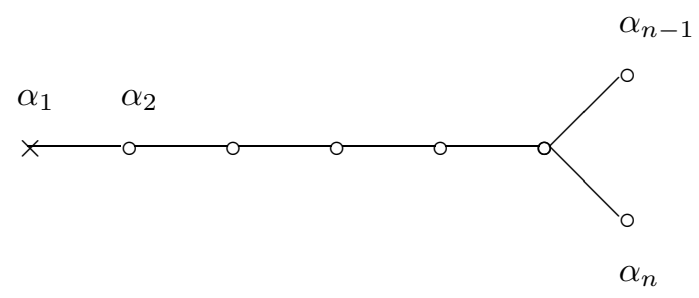

A maximal linear space $\mathbb{P}^{n-1}$ in $Q^{2(n-1)}$ corresponds to the element $w=$ $\sigma_{1} \cdots \sigma_{n-2} \sigma_{n-1}$ or the element $\tau=\sigma_{1} \cdots \sigma_{n-2} \sigma_{n}$ in $W^{P}$. Here, $\sigma_{i}$ stands for the reflection with respect to the simple root $\alpha_{i}$.

Consider the Schubert differential system $\mathcal{B}_{w}$ and Schur differential system $\mathcal{R}_{w}$ for the Schubert variety $X_{w}$. Then

$$
\begin{aligned}
\Delta(w) & =\left\{\alpha_{1}, \alpha_{1}+\alpha_{2}, \cdots, \alpha_{1}+\alpha_{2}+\cdots+\alpha_{n-2}+\alpha_{n-1}\right\} \\
D & =\left\{\lambda_{1}:=\alpha_{1}+2 \alpha_{2}+\cdots+2 \alpha_{n-2}+\alpha_{n-1}+\alpha_{n}\right\}
\end{aligned}
$$

and thus $s_{\sigma_{\gamma}\left(\lambda_{1}\right)}=x_{\lambda_{1}}$. We will show that

$$
\varphi_{\lambda_{1}}^{k}=x_{\lambda_{1}} \wedge x_{\alpha_{1}+\alpha_{2}} \wedge \cdots \wedge x_{\alpha_{1}+\alpha_{2}+\cdots+\alpha_{n-2}+\alpha_{n-1}}
$$

is contained in $\mathbf{I}_{\tau}=\left(\bigwedge^{n-1} \mathfrak{m}\right)^{\rho-\tau \rho}$. 
The lowest weight vector of $\mathbf{I}_{\tau}$ is given by

$$
e_{\Delta(\tau)}=x_{\alpha_{1}} \wedge x_{\alpha_{1}+\alpha_{2}} \wedge \cdots \wedge x_{\alpha_{1}+\alpha_{2}+\cdots+\alpha_{n-2}} \wedge x_{\alpha_{1}+\alpha_{2}+\cdots \alpha_{n-2}+\alpha_{n}} .
$$

The action by the root group $U_{\alpha_{2}+\cdots+\alpha_{n-2}+\alpha_{n-1}}$ does not change any vector in the multivector $e_{\Delta(\tau)}$ except the first and the last ones. Applying $U_{\alpha_{2}+\cdots+\alpha_{n-2}+\alpha_{n-1}}$ to $e_{\Delta(\tau)}$, we get an element

$$
x_{\alpha_{1}+\alpha_{2}+\cdots+\alpha_{n-2}+\alpha_{n-1}} \wedge x_{\alpha_{1}+\alpha_{2}} \wedge \cdots \wedge x_{\alpha_{1}+\alpha_{2}+\cdots+\alpha_{n-2}} \wedge x_{\lambda_{1}}
$$

in $\mathbf{I}_{\tau}$, which is a scalar multiple of $\varphi_{\lambda_{1}}^{k}$. Hence $\varphi_{\lambda_{1}}^{k}$ is not contained in $\mathbf{I}_{w}$, and, by Proposition 3.8, $\mathcal{B}_{w}$ is equal to $\mathcal{R}_{w}$.

General case. Let $w \in W^{P}$ correspond to the subgraph $\delta$ of type $A_{k}$ with $k=\ell(w)$. Since $\gamma$ is an end, we may choose the simple root system $\mathcal{S}=\left\{\alpha_{1}=\right.$ $\left.\gamma, \alpha_{2}, \cdots, \alpha_{n}\right\}$ such that $w$ is written by $\sigma_{1} \cdots \sigma_{k}$. Put $k_{0}=k_{G / P}$. Let $\delta_{0}$ be the subgraph of $\mathcal{D}(G)$ of type $A_{k_{0}}$ with two ends, $\gamma$ and the branch point (= the node connected to three other nodes).

Let $\epsilon$ be the subgraph of $\mathcal{D}(G)$ of type $D_{k_{0}+1}$ which contains $\delta_{0}$. We may assume that the simple roots are indexed in such a way that $\left\{\alpha_{1}, \cdots, \alpha_{k_{0}}, \alpha_{k_{0}+1}, \alpha_{k+1}\right\}$ is the set of simple roots in the subgraph $\epsilon$.

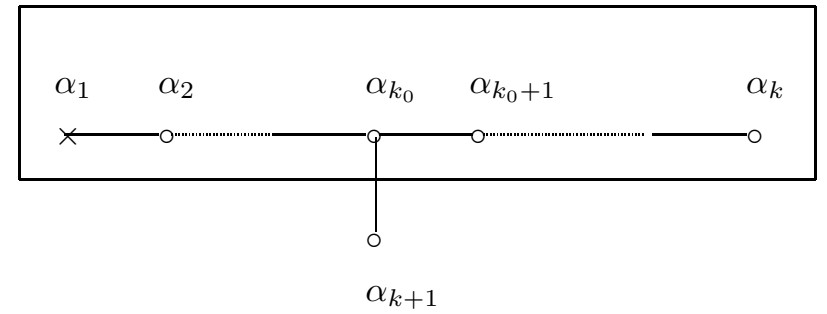

Put $\tau=\sigma_{1} \sigma_{2} \cdots \sigma_{k-1} \sigma_{k+1} \neq w \in W^{P}$. Since $\Delta(\tau)$ can be obtained from $\Delta(w)$ by replacing $\theta_{\delta}:=\alpha_{1}+\cdots+\alpha_{k}$ by $\theta_{\tau}:=\alpha_{1}+\cdots+\alpha_{k_{0}}+\alpha_{k+1}$, the lowest weight vector of $\mathbf{I}_{\tau}$ is given by

$$
e_{\Delta(\tau)}=x_{\alpha_{1}} \wedge \cdots \wedge \hat{x}_{\theta_{\delta}} \wedge x_{\theta_{\tau}}
$$

Here, we use the notation $\hat{x}_{\theta_{\delta}}$ to emphasize that $e_{\Delta(\tau)}$ does not have $x_{\theta_{\delta}}$ in its decomposition, compared to $e_{\Delta(w)}$ which can be considered as a base multivector. We will use this kind of notation to express various multivectors.

Write $\theta_{\delta}=\alpha_{1}+\beta_{1}$. Then $\beta_{1}=\alpha_{2}+\cdots+\alpha_{k}$ is a root in $\delta$, in particular, in $\mathfrak{l}_{w}$. The action of the root group $U_{\beta_{1}}$ does not change any vector in the multivector $e_{\Delta(\tau)}$ except $x_{\alpha_{1}}$ and $x_{\theta_{\tau}}$. Applying $U_{\beta_{1}}$ to $e_{\Delta(\tau)}$, we get an element

$$
\hat{x}_{\alpha_{1}} \wedge \cdots \wedge x_{\theta_{\delta}} \wedge x_{\theta_{\tau}+\beta_{1}} \in \mathbf{I}_{\tau} \text {. }
$$

We will show that for $\lambda \in D^{\prime \prime}, \varphi_{\lambda}^{k}=\hat{x}_{\alpha_{1}} \wedge \cdots \wedge x_{\theta_{\delta}} \wedge x_{\lambda}$ has a non-zero $\mathbf{I}_{\tau^{-}}$ component. We will prove it by showing that, after a successive adjoint action by the root vectors in $\mathfrak{p}_{0}$ to $\varphi_{\lambda}^{k}$, we get $\hat{x}_{\alpha_{1}} \wedge \cdots \wedge x_{\theta_{\delta}} \wedge x_{\theta_{\tau}+\beta_{1}} \in \mathbf{I}_{\tau}$.

First, assume that $\lambda$ is the minimal one in $D^{\prime \prime}$. Since the coefficient in $\alpha_{k+1}$ of any $\lambda \in D^{\prime \prime}$ is not zero, the $\lambda_{\mathbf{i}}$ for $\mathbf{i}=(1)$ (when $|N(\delta)|=1$ ) or $\mathbf{i}=(1,0)$ (when $|N(\delta)|=2)$ is the minimal one in $D^{\prime \prime}$. Then $\lambda-\left(\theta_{\tau}+\beta_{1}\right):=\beta_{2}$ is a root in $\mathfrak{p}_{0}$.

If $\beta_{2}$ is not a root in $\mathfrak{l}_{w}$, then for any $\alpha \in \Delta(w), \alpha-\beta_{2}$ is not a root. If $\beta_{2}$ is a root in $\mathfrak{l}_{w}$, then for some $\alpha \in \Delta(w), \alpha-\beta_{2}$ may be a root, but this $\alpha-\beta_{2}$ is another 
root in $\Delta(w)$. Since any $\lambda \in D^{\prime \prime}$ has coefficient 2 in $\alpha_{2}$, the coefficient in $\alpha_{2}$ of $\beta_{2}$ is zero and thus $\alpha_{1}+\beta_{2}$ is not a root. So $\alpha-\beta_{2}$ cannot be $\alpha_{1}$ for any $\alpha \in \Delta(w)$. Thus, in any case, $\hat{x}_{\alpha_{1}} \wedge \cdots \wedge\left[x_{-\beta_{2}}, x_{\alpha}\right] \wedge \cdots \wedge x_{\lambda}$ is zero for all $\alpha \in \Delta(w)$. So we get

$$
a d\left(x_{-\beta_{2}}\right) \varphi_{\lambda}^{k}= \pm \hat{x}_{\alpha_{1}} \wedge \cdots \wedge x_{\theta_{\delta}} \wedge x_{\theta_{\tau}+\beta_{1}} \in \mathbf{I}_{\tau} .
$$

Since $\operatorname{ad}\left(x_{-\beta_{2}}\right)$ preserves irreducible $\mathfrak{p}_{0}$-representation spaces in $\Lambda^{k} \mathfrak{m}, \varphi_{\lambda}^{k}$ has a no-zero $\mathbf{I}_{\tau}$-component.

$\lambda$ 's in $D^{\prime \prime}$ other than the minimal one, $\lambda_{\min }$, can be obtained from the minimal one by adding a root $\beta_{\lambda}$ in $\mathfrak{p}_{0}$ with a non-zero coefficient in a simple root in $N(\delta)$, successively. Then $\alpha-\beta_{\lambda}$ is not a root for any $\alpha \in \Delta(w)$. Thus we get

$$
\operatorname{ad}\left(x_{-\beta \lambda}\right) \varphi_{\lambda}^{k}=\hat{x}_{\alpha_{1}} \wedge \cdots \wedge x_{\theta_{\delta}} \wedge x_{\lambda_{\text {min }}} \notin \mathbf{I}_{w}
$$

and hence $\varphi_{\lambda}^{k} \notin \mathbf{I}_{w}$.

Now we consider the equality $B_{w}=R_{w}$ when the Schubert variety $X_{w}$ corresponds to a subgraph $\delta$ of $\mathcal{D}(G)$ of type $D_{\ell}$ or $C_{\ell}$. The proof will be almost the same, but the difference is that in this case, the highest weight $\theta_{\delta}$ is not obtained from $\alpha_{1}=\gamma$ by just adding one root $\beta_{1}$ but by adding two roots $\beta_{1}$ and $\beta_{2}$ successively. So we need to apply the adjoint actions more times to get an element in $\mathbf{I}_{\tau} \neq \mathbf{I}_{w}$.

Proposition 4.8. Let $G / P$ be a Hermitian symmetric space $\neq G r(m, n)$ associated to the simple root $\gamma$. Let $\delta$ be a subgraph of $\mathcal{D}(G)$ of type $D_{\ell}$ with $\gamma$ an extremal node or of type $C_{\ell}$ with $\gamma=\alpha_{\ell}$, and let $X_{w}$ be the corresponding Schubert variety. Then $\mathcal{B}_{w}$ is equal to $\mathcal{R}_{w}$.

We remark that the case of sub-Lagrangian Grasmmanians in the Lagrangian Grassmannian $L_{m}$ was proved in Lemma 14 of [B].

Proof. Let $\left\{\alpha_{1}, \cdots, \alpha_{\ell}\right\}$ be the simple roots in $\delta$ with $\alpha_{1}=\gamma$. Then $w$ is the longest element in the subgroup of the Weyl group generated by the reflections $\sigma_{i}=\sigma_{\alpha_{i}}$, $i=1, \cdots, \ell$. Write $w=\sigma_{i_{1}} \cdots \sigma_{i_{k}}$, where $k=\ell(w)$. Let $\alpha_{\ell+1}$ be the simple root in $N(\delta)$. Put $\tau=\sigma_{i_{1}} \cdots \sigma_{i_{k-1}} \sigma_{\ell+1}$. Then $\ell(\tau)=\ell(w)$ and

$$
\Delta(\tau)=\Delta(w)-\left\{\theta_{\delta}\right\} \cup\left\{\theta_{\tau}\right\},
$$

where $\theta_{\delta}$ is the maximal root in the root system of $\delta$ and $\theta_{\tau}$ is the maximal root in the root system of the subgraph $\epsilon$ of type $A_{\ell}$ containing $\alpha_{1}$ and $\alpha_{\ell+1}$. After rearranging the indexes of the simple roots, we may assume that $\left\{\alpha_{1}, \cdots, \alpha_{\ell-1}, \alpha_{\ell+1}\right\}$ is the simple root system of $\epsilon$. Then $\theta_{\tau}=\alpha_{1}+\cdots+\alpha_{\ell-1}+\alpha_{\ell+1}$.

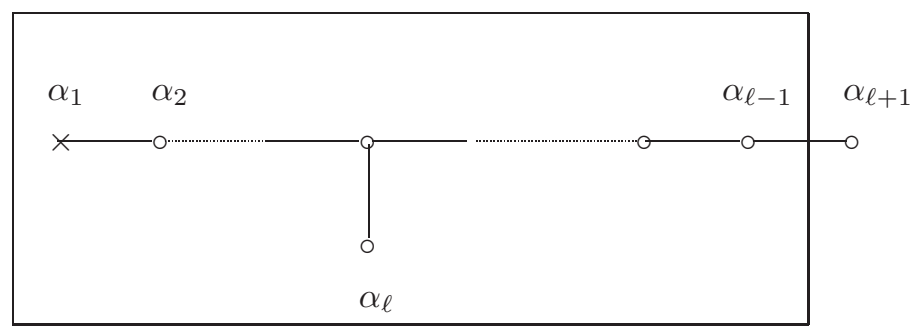

We will show that for $\lambda \in D^{\prime \prime}, \varphi_{\lambda}^{k}=\hat{x}_{\alpha_{1}} \wedge \cdots \wedge x_{\theta_{\delta}} \wedge x_{\lambda}$ has a non-zero $\mathbf{I}_{\tau^{-}}$ component. By the same argument as in the proof of Proposition 4.7, it suffices 
to show this for the minimal $\lambda_{1}$ in $D^{\prime \prime}$. (In this case, since $|N(\delta)|=1, \lambda_{1}$ is the minimal one.)

Write $\theta_{\delta}$ as the sum $\alpha_{1}+\beta_{1}+\beta_{2}$, where $\beta_{1}:=\alpha_{2}+\cdots+\alpha_{\ell-2}$ and $\beta_{2}:=$ $\alpha_{2}+\cdots+\alpha_{\ell-1}+\alpha_{\ell}$ are roots in $\delta$, in particular, in $\mathfrak{l}_{w}$. Let $\lambda=\lambda_{1}$ be the minimal one in $D^{\prime \prime}$. Then one can check that $\lambda-\beta_{1}$ is not a root.

Write $\lambda=\theta_{\tau}+\beta_{3}+\beta_{4}$. Here, $\beta_{3}$ is $\lambda-\theta_{\tau}$, if $\lambda-\theta_{\tau}$ is a root, and $\beta_{3}$ is the maximal root among the roots with each coefficient $\leq$ each coefficient in $\lambda-\theta_{\tau}$, otherwise. Then $\beta_{4}=0$ in the former case and $\beta_{4}=\lambda-\theta_{\tau}-\beta_{3}$ in the latter case.

Note that $\beta_{3}$ has coefficient 1 in $\alpha_{\ell}$, and $\beta_{4}$ is a root in $\mathfrak{p}_{0}$ with coefficient 1 in $\alpha_{\ell}$ and zero coefficient in $\alpha_{2}$ when $\lambda-\theta_{\tau}$ is not a root. So neither $\theta_{\tau}-\beta_{2}+\beta_{3}$ nor $\theta_{\tau}-\beta_{2}+\beta_{4}$ is a root. (For, the coefficient of $\theta_{\tau}-\beta_{2}+\beta_{3}$ and $\theta_{\tau}-\beta_{2}+\beta_{4}$ in $\alpha_{\ell}$ is zero, and any root with zero coefficient in $\alpha_{\ell}$ has all coefficients $\leq 1$.)

Since $\lambda-\beta_{1}$ is not a root, we get

$$
\operatorname{ad}\left(x_{-\beta_{1}}\right)\left(\varphi_{\lambda}^{k}\right)= \pm x_{\alpha_{1}} \wedge \cdots \wedge \hat{x}_{\alpha_{1}+\beta_{1}} \wedge \cdots \wedge x_{\theta_{\delta}} \wedge x_{\lambda}
$$

so that

$$
\begin{aligned}
& \operatorname{ad}\left(x_{-\beta_{2}}\right) \operatorname{ad}\left(x_{-\beta_{1}}\right)\left(\varphi_{\lambda}^{k}\right) \\
= & \pm x_{\alpha_{1}} \wedge \cdots \wedge \hat{x}_{\theta_{\delta}} \wedge x_{\lambda} \pm x_{\alpha_{1}} \wedge \cdots \wedge \hat{x}_{\alpha_{1}+\beta_{1}} \wedge \cdots \wedge x_{\theta_{\delta}} \wedge\left[x_{-\beta_{2}}, x_{\lambda}\right] .
\end{aligned}
$$

Since $\theta_{\delta}$ is the maximal root in $\delta$ and $\lambda-\beta_{2}-\beta_{3}=\theta_{\tau}-\beta_{2}+\beta_{4}$ is not a root, we get

$$
\operatorname{ad}\left(x_{-\beta_{3}}\right) \operatorname{ad}\left(x_{-\beta_{2}}\right) \operatorname{ad}\left(x_{-\beta_{1}}\right)\left(\varphi_{\lambda}^{k}\right)= \pm x_{\alpha_{1}} \wedge \cdots \wedge \hat{x}_{\theta_{\delta}} \wedge x_{\theta_{\tau}+\beta_{4}}+\mathbf{v},
$$

where $\mathbf{v}$ is $\pm x_{\alpha_{1}} \wedge \cdots \wedge \hat{x}_{\alpha_{1}+\beta_{1}+\beta_{3}} \wedge \cdots \wedge x_{\theta_{\delta}} \wedge\left[x_{-\beta_{2}}, x_{\lambda}\right]$ if $\alpha_{1}+\beta_{1}+\beta_{3}$ is a root in $\delta$, and is zero otherwise. Since $\lambda-\beta_{2}-\beta_{4}=\theta_{\tau}-\beta_{2}+\beta_{3}$ is not a root, we get

$$
\operatorname{ad}\left(x_{-\beta_{4}}\right) \operatorname{ad}\left(x_{-\beta_{3}}\right) \operatorname{ad}\left(x_{-\beta_{2}}\right) a d\left(x_{-\beta_{1}}\right)\left(\varphi_{\lambda}^{k}\right)= \pm x_{\alpha_{1}} \wedge \cdots \wedge \hat{x}_{\theta_{\delta}} \wedge x_{\theta_{\tau}}+\mathbf{v}^{\prime},
$$

where $\mathbf{v}^{\prime}$ is a decomposable $k$-vector.

In any case, since $e_{\Delta(\tau)}=x_{\alpha_{1}} \wedge \cdots \wedge \hat{x}_{\theta_{\delta}} \wedge x_{\theta_{\tau}}$ is a $k$-vector in $\mathbf{I}_{\tau}$ and $x_{-\beta_{4}}$, $x_{-\beta_{3}}, x_{-\beta_{2}}$ and $x_{-\beta_{1}}$ are root vectors of $\mathfrak{p}_{0}, \varphi_{\lambda}^{k}$ has a non-zero $\mathbf{I}_{\tau}$-component. Hence $\varphi_{\lambda}^{k} \notin \mathbf{I}_{w}$ for any $\lambda \in D^{\prime \prime}$, and thus $B_{w}=R_{w}$ by Proposition 3.8.

The case when $\delta$ is of type $\left(C_{\ell}, \alpha_{\ell}\right)$ can be proved in a similar way by taking $\tau=\sigma_{1} \cdots \sigma_{i_{k-1}} \sigma_{\ell+1}$ after re-indexing the simple roots in the following way:

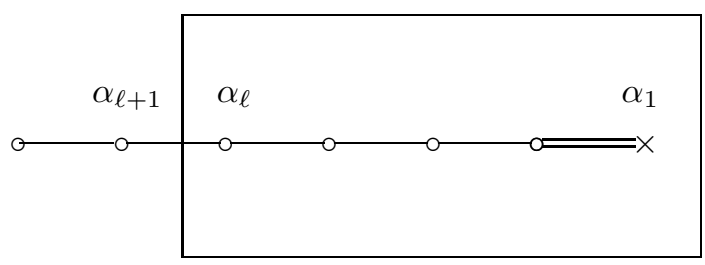

Then $\Delta(\tau)=\Delta(w)-\left\{\theta_{\delta}\right\} \cap\left\{\theta_{\tau}\right\}, \theta_{\tau}=\alpha_{1}+\cdots+\alpha_{\ell+1}$ and $\theta_{\delta}=\alpha_{1}+\beta_{1}+\beta_{2}$, where $\beta_{1}=\beta_{2}=\alpha_{2}+\cdots+\alpha_{\ell}$.

4.4. Conclusion. Recall that $k_{G / P}$ is defined by the length of the chain with extremal node $\gamma$ and the branch point (Section 4.1). 
Theorem 4.9. Let $G / P$ be a Hermitian symmetric space $\neq Q^{2 n-1}$. Let $\delta$ be a subgraph of $\mathcal{D}(G)$ of type $A_{k}$ with $\gamma$ an extremal node and let $X_{w}$ be the corresponding Schubert variety. Suppose that $k>k_{G / P}$. Then a subvariety $X$ of $G / P$ with the homology class $[X]=r\left[X_{w}\right]$ is contained in a maximal linear space in $G / P$. In particular, the cycle space $\mathcal{Z}_{k}\left(G / P,\left[X_{w}\right]\right)$ consists of Schubert varieties of type $w$.

If $\delta$ is a maximal chain, then $X_{w}$ is Schur rigid.

Proof. By Proposition 4.7, any integral variety of $\mathcal{R}_{w}$ is an integral variety of $\mathcal{B}_{w}$ if $k>k_{G / P}$. By Proposition 4.1, an integral variety of $\mathcal{B}_{w}$ is contained in a maximal linear space if $k>k_{G / P}$. Since a subvariety of a linear space of degree 1 is again a linear space, the cycle space $\mathcal{Z}_{k}\left(G / P,\left[X_{w}\right]\right)$ consists of Schubert varieties of type $w$.

Theorem 4.10. Let $G / P$ be a Hermitian symmetric space associated to the simple root $\gamma$. Let $\delta$ be a subgraph of $\mathcal{D}(G)$, either

(1) of type $A_{k}$ with $\gamma$ non-extremal node or

(2) of type $D_{\ell}$ with $\gamma$ an extremal node or

(3) of type $C_{\ell}$ with $\gamma=\alpha_{\ell}$.

Then the corresponding Schubert variety $X_{w}$ is Schur rigid.

Proof. By Proposition 2.10, it suffices to prove the Schubert rigidity and the equality $B_{w}=R_{w}$. The former is given by Proposition 4.3 and the latter is given by Proposition 4.6 for case (1) and by Proposition 4.8 for cases (2) and (3).

By Proposition 2.12, Schubert varieties considered in Theorem 4.9 and Theorem 4.10 are all the smooth Schubert varieties in Hermitian symmetric spaces. So we prove

Main Theorem. Let $G / P$ be a Hermitian symmetric space other than an odddimensional quadric. Then any smooth Schubert variety $X_{w}$ in $G / P$ is Schur rigid except when $X_{w}$ is a non-maximal linear space in $G / P$. Here, we consider $G / P$ as a projective variety by the minimal equivariant embedding $G / P \subset \mathbb{P}(V)$.

\section{ACKNOWLEDGMENTS}

I thank the referee for several suggestions which improved this paper. This work was supported by the Post-Doctorial Fellowship Program of Korea Science and Engineering Foundation (KOSEF).

\section{REFERENCES}

[B] R. Bryant, Rigidity and quasi-rigidity of extremal cycles in compact Hermitian symmetric spaces, to appear in Annals of Mathematics Studies 153, Princeton University Press.

[BE] R. Baston and M. Eastwood, The Penrose Transform Its interaction with representation theory, Oxford Science Publication, 1989 MR 1038279 (92j:32112)

[BP] M. Brion and P. Polo, Generic singularities of certain Schubert varieties, Math. Z., 231, 301-324 (1999) MR1703350 (2000f:14078)

$[\mathrm{CH}]$ I. Choe and J. Hong, Integral varieties of the canonical cone structure on $G / P$, Math. Ann. 329, 629-652 (2004) MR2076680 (2005e:32040)

[F] W. Fulton, Young tableaux. With application to representation theory and geometry, London Mathematical Society Student Texts, 35, Cambridge University Press, 1997 MR.1464693 (99f:05119)

[FH] W. Fulton and J. Harris, Representation Theory; A First Course, Springer-Verlag, 1991 MR.1153249 (93a:20069) 
[G] A. B. Goncharov, Generalized conformal structures on manifolds, Selecta Mathematica Sovietica, vol. 6, No. 4, 1987. MR0925263 (89e:53050)

[K1] B. Kostant, Lie algebra cohomology and the generalized Borel-Weil theorem, Ann. of Math. (2) 74, No. 2, 329-387 (1961). MR0142696 (26:265)

[K2] B. Kostant, Lie algebra cohomology and generalized Schubert cells, Ann. of Math. (2) 77, No. 1, 72-144 (1963). MR0142697(26:266)

[LW] V. Lakshmibai and J. Weyman, Multiplicities of points on a Schubert variety in a minuscule $G / P$, Advances in Mathematics 84, 179-208 (1990) MR1080976 (92a:14058)

[W] M. Walters, Geometry and uniqueness of some extreme subvarieties in complex Grassmannians, Ph.D. thesis, University of Michigan, 1997.

Research Institute of Mathematics, Seoul National University, San 56-1 Sinrim-dong KWanak-Gu, Seoul, 151-747 Korea

E-mail address: jhhong@math.snu.ac.kr 